
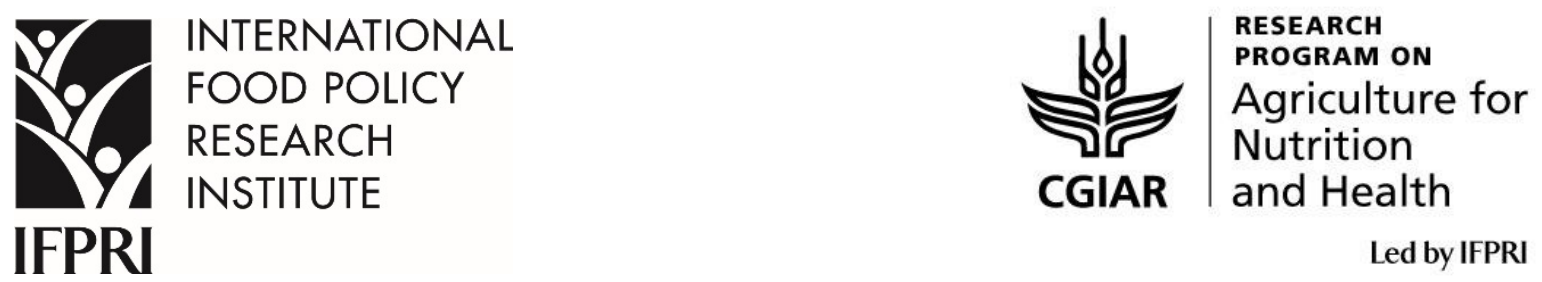

Led by IFPRI

IFPRI Discussion Paper 01874

October 2019

\title{
Accounting for the Rapid Reduction of Child Stunting in Tanzania over 2005-2016
}

\author{
Derek Headey \\ Jessica Heckert \\ Biram Ndiaye \\ Mauro Brero \\ Vincent Assey
}

Poverty, Health, and Nutrition Division 


\section{INTERNATIONAL FOOD POLICY RESEARCH INSTITUTE}

The International Food Policy Research Institute (IFPRI), established in 1975, provides research-based policy solutions to sustainably reduce poverty and end hunger and malnutrition. IFPRI's strategic research aims to foster a climate-resilient and sustainable food supply; promote healthy diets and nutrition for all; build inclusive and efficient markets, trade systems, and food industries; transform agricultural and rural economies; and strengthen institutions and governance. Gender is integrated in all the Institute's work. Partnerships, communications, capacity strengthening, and data and knowledge management are essential components to translate IFPRI's research from action to impact. The Institute's regional and country programs play a critical role in responding to demand for food policy research and in delivering holistic support for country-led development. IFPRI collaborates with partners around the world.

\section{AUTHORS}

Derek Headey (d.headey@cgiar.org) is a Senior Research Fellow in the Poverty, Health, and Nutrition Division of The International Food Policy Research Institute (IFPRI), Washington DC.

Jessica Heckert (j.heckert@cgiar.org) is a Research Fellow in the Poverty, Health, and Nutrition Division of IFPR, Washington, DC.

Biram Ndiaye (bindiaye@, unicef.org) is Chief of Nutrition at UNICEF South Sudan Country Office, Juba, South Sudan.

Mauro Brero (mbrero@unicef.org) is Chief of Nutrition at UNICEF Tanzania Country Office, Dar es Salam, Tanzania.

Vincent Assey (vdassey@gmail.com) is the Managing Director of the Tanzania Food and Nutrition Center (TFNC), Dar es Salaam, Tanzania.

\footnotetext{
Notices

${ }^{1}$ IFPRI Discussion Papers contain preliminary material and research results and are circulated in order to stimulate discussion and critical comment. They have not been subject to a formal external review via IFPRI's Publications Review Committee. Any opinions stated herein are those of the author(s) and are not necessarily representative of or endorsed by IFPRI.

${ }^{2}$ The boundaries and names shown and the designations used on the map(s) herein do not imply official endorsement or acceptance by the International Food Policy Research Institute (IFPRI) or its partners and contributors.

${ }^{3}$ Copyright remains with the authors. The authors are free to proceed, without further IFPRI permission, to publish this paper, or any revised version of it, in outlets such as journals, books, and other publications.
} 


\section{Contents}

ABSTRACT iv

ACKNOWLEDGMENTS vi

ACRONYMS vii

1. Introduction 1

2. Data and Methods $\quad 4$

$\begin{array}{ll}2.1 \text { Data } & 4\end{array}$

2.2 Methods $\quad 9$

2.2.1 Descriptive analysis $\quad 9$

2.2.2 Regression analysis $\quad 9$

2.2.3 Decomposition analysis 11

2.2.4 Projection analysis $\quad 11$

3. Descriptive Results 12

3.1 Trends in stunting $\quad 12$

3.2 Trends in potential determinants of stunting 20

4. Regression analysis 26

4.1 Main regression results 26

4.2 Robustness tests $\quad 29$

4.3 An extension to consider the role of child dietary diversity in reducing stunting 33

5. Past and future drivers of stunting reduction 41

5.1 Using linear decompositions at means to assess drivers of stunting reduction over 2005-2015 41

5.2 Potential drivers of future stunting reduction in Tanzania: 2015-2025 43

6. Conclusions $\quad 47$

REFERENCES $\quad 49$ 


\begin{abstract}
This research was undertaken in order to understand what factors have been driving stunting reduction in Tanzania over the recent past (2005-2015), and what can further accelerate progress against undernutrition in the near future (2015-2025). Chronic undernutrition in early childhood - often indicated by stunting has highly detrimental consequences for long-term physical and cognitive development, school attendance and performance, and labor productivity and wages in adulthood. Understanding how countries have been able to successfully reduce stunting prevalence, and how they can accelerate this success in the future, are therefore critically important research questions. During the past decade, Tanzania has experienced rapid change in the nutrition status of children 0-5 years, particularly since 2010. Stunting declining from 44.3\% in $2004-05$ to $42.0 \%$ in 2010 , before dropping sharply to $34.4 \%$ in $2015-16$, a decline of 0.9 percentage points per year. This encouraging trend begs the main research question motivating this paper: what explains this progress?

We address this question with a quantitative approach built upon both parametric and non-parametric regression techniques, and a simple linear regression decomposition at means. Previous studies applying this approach in South Asia have demonstrated the paramount importance of improvements in household wealth, parental education, maternal and child health care, and water, sanitation and hygiene (WASH). However, a novel contribution of the study is the consideration of infectious diseases, including malaria and HIV/AIDS: both diseases were very common in 2005, yet both saw dramatic improvements in prevention and treatment efforts in subsequent years. We show that proxies for the prevention/treatment of these diseases account for the largest share of the predicted reduction in stunting over 2005-16, that prevention of both diseases is associated with improvements in early childhood nutrition (suggestive of improvements in birth size and growth in early infancy), and that the apparent effects of these public health efforts are larger in regions where the diseases are more prevalent. In contrast, the apparent effects of household wealth, parental education, maternal and child health care and WASH are more important in explaining stunting reduction among older children.
\end{abstract}


Finally, we go beyond historical decompositions by using the regression results to project alternative stunting reduction scenarios to 2025. If trends over 2005-2015 were to continue, Tanzania would fail to achieve the WHA target of a $40 \%$ reduction in stunting by 2025 . However, an accelerated socio-economic development scenario - in which Tanzania achieves faster progress in a wide range of sectors, including improved child feeding - could achieve the WHA target and see stunting rates fall by a further 14 percentage points by 2025. These results reaffirm that solving stunting requires rapid and coordinated progress in multiple sectors, including nutrition-specific actions and a wide range of nutrition-sensitive actions.

Keywords: undernutrition; stunting; Tanzania; HIV/AIDS; malaria. 


\section{ACKNOWLEDGMENTS}

These studies were requested by Tanzania's High level Steering Committee on Nutrition after its review of the Tanzania Demographic Health Survey 2015/16 in order to better understand the changes in nutritional status among women and children in Tanzania. TFNC and UNICEF through funding from Irish Aid engaged the Poverty, Health, and Nutrition Division of the International Food Policy Research Institute to conduct this study. Findings were presented and validated to the Tanzania Nutrition Community including Prime Minister's Office (PMO), Ministry of Health Community Development Gander Elderly and Children (MOHCDGEC), President' Office - Regional Authorities and Local Government (PORALG), National Bureau of Statistics (NBS) as well as representatives from academia, civil society organizations, United Nations and development partners. The authors would like to thank participants at these workshops, Wahid Quabili for assistance on data preparation, and Giordano Palloni for contributions to the analysis.

This work was undertaken as part of the CGIAR Research Program on Agriculture for Nutrition and Health (A4NH). 


\section{ACRONYMS}

AIS

ART

DDS

DHS

HAZ

HIV/AIDS

JMP/WHO

MDD

MTCT

WASH

WHA

WHO
AIDS Indicator Surveys

Anti-retroviral therapy

Dietary diversity score

Demographic Health Surveys

Height-for-age Z score

Human immunodeficiency virus/ Acquired immunodeficiency syndrome

Joint Monitoring Program/World Health Organization

Minimum dietary diversity

Maternal to child transmission

Water, sanitation and hygiene

World Health Assembly

World Health Organization 


\section{INTRODUCTION}

Undernutrition in early childhood has highly detrimental consequences for long-term physical and cognitive development, schooling attendance and performance, and labor productivity and wages in adulthood (Hoddinott, Alderman, Behrman, Haddad, \& Horton 2013). During the past decade, Tanzania has experienced rapid change in the nutrition status of children 0-5 years, with official estimates of stunting (height-for-age $\mathrm{z}$ score $<-2$ standard deviations below the mean) declining from $44.3 \%$ in 2005 to

$34.4 \%$ in 2016, a decline of 0.9 percentage points per year. This encouraging trend begs the main research question motivating this paper: what explains this progress?

Addressing such a question is challenging. As is well known, nutritional change is driven by improvements in multiple sectors, including the productive sectors of the economy, as well as social sectors such as education, health/nutrition, WASH, and family planning. Identifying the contribution of these sectors is challenging because experimental designs are generally not applicable to national-level data, and are, in any case, not well suited to identifying the impacts of changes across multiple sectors. Qualitative approaches, as applied elsewhere in the literature on nutritional success stories (Gillespie \& van den Bold 2017; Heaver \& Kachondam 2002), are essential for understanding the policy processes underlying national level change in nutrition but stop short of identifying and quantifying which policies and programs have made a substantive difference to nutritional change. Such analyses also tend to be more focused on nutrition-specific interventions, even though most nutritional change is typically driven by nutrition-sensitive factors, such as income growth, improved in education, WASH, demographic change, and various public health interventions. On the other hand, many quantitative observational analyses of nutrition have identified plausible determinants of nutritional differences across children but stopped short of conducting more dynamic analyses of which factors may be driving nutritional change over time.

In this study we apply an alternative approach to understanding the "drivers" of nutritional change in Tanzania. This approach is quantitative and dynamic, although it builds on the more 
qualitative analysis presented in Chapter 2 of this report. Moreover, the quantitative approach is not without limitations. We build on the methods developed in Headey, et al. (2015), Headey and Hoddinott (2014), and Headey, Hoddinott, and Park (2016). The essence of this approach is to pool multiple rounds of data together, to analyze trends in stunting and various explanatory factors, and then use a pooled regression analysis to implement a decomposition of which factors best account for changes in nutrition outcomes over time. This approach is not without its limitations (see below), but it does offer a transparent and agnostic means of exploring the potential importance of developments in a wide range of nutrition-sensitive sectors. More rigorous experimental analyses cannot do this, and qualitative approaches to understanding success stories tend to focus more on specific sectors and preclude the possibility of quantifying or formally testing for the importance of different sectors.

The context of this analysis, Tanzania, is particularly interesting, but also challenging. Like other countries experiencing relatively rapid improvements in nutrition, it is unlikely that any single factor explains nutritional change in Tanzania. The Tanzanian economy improved substantially over 2005-2016, bringing about improvements in household wealth, but Tanzania's economic performance has been solid rather than spectacular, and wealth gains alone are unlikely to explain the country's recent nutritional success. In this paper we conjecture that improvements in the health environment - particularly combating widespread, highly infectious and highly dangerous infectious diseases such as HIV/AIDS and malaria - offer an important and novel explanation of nutritional improvements in young children. Scarcely any previous research has documented impacts of large scale efforts to prevent or treat HIV/AIDS or malaria on child nutrition, and this marks a novel contribution of our analysis.

A second novel contribution of our study is to look forward as well as back. While Tanzania has made impressive progress against child stunting in recent years, the speed of stunting change over 2005-2015 is not sufficient to meet the World Health Assembly target of reducing stunting by 40\% over 2015 to 2025 . 
Moreover, some of the quick wins from prevention and treatment of infectious diseases may be hard to further sustain, and progress on other fronts over 2005-2015 was very limited. In particular, we show that children's diets have failed to diversify and require substantial improvement (a likely explanation of why stunting rates increased rapidly in the 6-23 month window), while family planning outcomes (birth intervals, teenage pregnancies) also failed to improve over 2005-2015. In other areas, Tanzania saw modest developments over this period (wealth, basic health services, education, WASH) that need to be accelerated still further. In this paper extend the decomposition approach to forecast potential stunting reductions in the future to compare a baseline scenario (where nutrition-sensitive development proceed at the same rate as they did over 2005-2015) to an accelerated socio-economic development scenario (where progress accelerates on multiple fronts). We show that only this accelerated development scenario can achieve the WHA 2025 target, suggesting the need for more proactive and more effective multisectoral coordination and development.

The remainder of this paper is structured as follows. Section 2 describes our data and methods. Section 3 offers a descriptive view of changes in nutrition and its underlying determinants in Tanzania using conventional descriptive statistics, maps and non-parametric regressions. Section 4 presents our core regression results, and various robustness tests. Section 5 presents decomposition results to assess the major putative drivers of stunting reduction over 2005-2015, as well as an extension to examine possible nutritional change scenarios over 2015-2025. Section 6 concludes. 


\section{DATA AND METHODS}

To examine the drivers of nutritional change, a regression-decomposition approach is used. This approach, first applied to understanding trends in the decline in stunting by Headey et al. (2015) to Bangladesh, and subsequently to other South Asian and sub-African countries (Headey 2014; Headey et al. 2016; Headey \& Hoddinott 2015), is based on four simple steps:

(1) Preparing multiple rounds of Demographic Health Survey (DHS) data so that all variables are strictly standardized across surveys.

(2) Analyzing trends in nutrition outcomes and its determinants, including age-disaggregated nutrition outcomes, and trends in the survey-weighted means of the explanatory variables.

(3) Conducting multivariate regression analysis to see which factors have statistically significant associations with the nutrition outcome(s).

(4) Using the observed changes in the means of the explanatory variables (Step 2) and the regression coefficients (Step 3) to estimate the predicted historical change in nutrition, so as to assess which factors account for historical changes in nutrition, and whether the model as a whole accurately predicts nutritional change over time.

In this section we describe these steps in more detail.

\subsection{Data}

In the first step, nationally representative data from the 2004-2005, 2010, and 2015-2016 Tanzania DHS were merged together to satisfy the bulk of our data requirements. The DHS is a rigorous multicountry survey instruments implemented by ICF International (2017) and the Government of Tanzania. However, different rounds of the DHS change over time, and the first step in our analysis was to construct a pooled multi-round dataset with consistently defined indicators.

To do so, we first constructed consistent regional identifiers. The DHS are nationally representative, but also representative of rural/urban Tanzania and various subnational regions. However, since the 
subnational representativeness of the DHS has changed over time, so to ensure consistency and facilitate meaningful spatial comparisons, we defined two levels of aggregation. First, to explore subnational trends we defined eight ecological zones. Second, we used the 26 original regions of Tanzania present in 2005 (prior to several new regions being formed in 2012) to define subnational fixed effects in our regression models.

The primary outcome indicators are the height-for-age $\mathrm{Z}$ score, which measure height relative to the 2006 WHO Growth Reference Standards (WHO, 2006), and stunting, measured as HAZ $<2$. In robustness tests we also look at severe stunting, measured as $\mathrm{HAZ}<-3$.

The selection of putative drivers of change in nutrition outcomes among children was largely informed by the UNICEF (1990) and Lancet nutrition frameworks (Black et al., 2013), and previous regressiondecomposition studies of nutritional change in developing countries. These variables are described in Table 2.1 below. Most are derived from the Tanzania DHS, although as we describe below, HIV programming proxies, HIV prevalence and malaria prevalence were derived from other sources.

Most of the explanatory variables forced from the DHS are standard and present in previous regressiondecomposition analyses. Indicators are measured at different units of analysis: child, mother, household, village and region. We follow previous studies in constructing a household asset index using principal components analysis, with the weights on each asset consistent across DHS rounds. We use years of secondary education for mothers and fathers because previous research has found that only secondary education contributes to improved nutrition among children (Alderman \& Headey 2017), and because preliminary results suggested no significant impact of primary education. We follow the JMP/WHO definition of improved drinking water and sanitation, as described in Gunther and Fink (2010), but we also experimented with alternative measures, including cluster-level open defecation, which was not statistically significant in our main models.

In terms of health service indicators, we measure access to medical facilities at the cluster level; specifically, the share of children born surveyed in a given survey cluster (or "village") that were born in 
a medical facility. We interpret this as a general proxy for access to health facilities, rather than a specific causal pathway linking medical facility births to child growth. We also use a more specific measure of antenatal care - whether a mother had her blood tested during pregnancy - as a potentially important indicator of whether a mother could be treated for a range of health problems that are widely prevalent in Tanzania, including malaria, helminth infections, anemia and HIV/AIDS.

Finally, we considered a range of demographic variables of potential importance to child growth: adolescent mothers (maternal age at birth $<20$ years), maternal BMI and short stature (maternal height $<145 \mathrm{~cm}$ ), whether the child is female, and whether the survey cluster is rural, as defined by national definitions.

While the indicators above are quite standard, we also hypothesized that HIV/AIDS and malaria might be important determinants of stunting in Tanzania, and major public health campaigns to treat and prevent these diseases could at least partially account for the rapid reduction of stunting in Tanzania.

The impacts of malaria on child growth are not well understood but could operate both prenatally and postnatally. There is some evidence that maternal malaria is associated with lower birthweight, and perhaps by extension birth size, or neonatal stunting (Eisele et al., 2012; Unger et al., 2016). The effects of malaria on postnatal growth are somewhat unclear, although repeated infections are expected to divert biological resources away from growth towards fighting infection. However, an authoritative literature review found the literature on malaria and child growth to be remarkably small, quite flawed and yielding inconsistent evidence (Black and Jackson, 2017). ${ }^{1}$

To capture malaria prevention efforts, we measure household bed net ownership at the community rather than household level, since there are clear externalities to a neighbor's use of bed nets because malaria spreads via human hosts, as well as livestock. However, one would expect bednet ownership to have more impact in areas prone to malaria, and malaria prevalence is not recorded in the DHS. To measure baseline

\footnotetext{
${ }^{1}$ A handful of observational studies found some impact of malaria on child growth, but several randomized control trials delivering malaria treatment found no impacts on stunting, although these studies also suffered from important designed flaws.
} 
malaria prevalence in a region we merged a Geographic Information Systems indicator of malaria prevalence among children 2-9 years to each region. These data come from the Malaria Atlas Project (Bhatt et al., 2015).

Measurement of HIV/AIDS programming is even more challenging, but there are certainly strong biological and socioeconomic mechanisms linking maternal HIV/AIDS to child nutrition. First, children can become HIV-infected through direct mother-to-child transmission (MTCT) of HIV, and pediatric HIV/AIDS can lead to poor development in utero, but also postnatal growth and development problems due to increased infections, loss of appetite, reduced nutrient absorption and hence poorer growth among children (Lartey, et al., 2014; Omoni et al., 2017). Second, socioeconomic channels include parental fatigue, illness or death, leading to inadequate child care or loss of household income. Third, treatments for HIV/AIDS, including ART, can also have side effects that may influence child growth in utero or postnatally even if the child remains uninfected by HIV, although these effects are not well understood (Lane et al. 2019).

Unfortunately, the Tanzanian DHS does not collect HIV/AIDS treatment indicators, meaning that we required proxy measures from other sources. We therefore took various indicators from the AIDS Indicator Surveys (AIS), which are part of the DHS family of surveys, but which took place 2-3 years prior to the DHS. Because we cannot match data at the household or village level, we include the mean of each indicator for all 26 regions, linking the 2003-04 AIS to the 2005 DHS, the 2007-08 AIS to the 2010 DHS, and the 2011-12 AIS to the 2015 DHS. While this lagged structure could be potentially problematic in other contexts, the cumulative nature of child growth arguably makes lagged indicators attractive, because maternal HIV/AIDS and treatment status in utero and early infancy are likely more relevant than at older ages in which children are at lower risk of MTCT.

The main HIV/AIDS programming proxy we focus on is the share of mothers 15-49 years of age in a region who know that drugs (i.e. anti-retroviral therapy, ART) can be used to prevent MTCT. This indicator is relevant to child stunting because maternal knowledge of ART is essential for its uptake, such 
that we expect this indicator to be a good proxy for actual use of ART by pregnant mothers. We also test robustness using the share of adults in a region who were tested for HIV/AIDS in the 12 months prior to the AIS survey, since HIV/AIDS testing is a prerequisite for ART. The limitations of these regional indicators are twofold. First, we have relatively few degrees of freedom (78 region-year observations), which could lead to imprecision in our regression estimates. Second, these indicators are only indirect proxies for access to ART, our main unobserved variable of interest. Our assumption is that efforts to extend ART involved increase ART knowledge/awareness as well as increased testing.

Table 2.1 Definitions of key variables used in the study

\begin{tabular}{|c|c|}
\hline Short name & Definition \\
\hline Stunting (HAZ<-2) & Height-for-age Z-score of -2 or less \\
\hline Child: female $(0 / 1)$ & Child is female \\
\hline Child: birth interval $<18 \mathrm{~m}(0 / 1)$ & Birth interval less than 18 months \\
\hline Mother: teen pregnancy $(0 / 1)$ & Mother $<20$ years old \\
\hline Mother: BMI score & Maternal body mass index score \\
\hline Mother: short $(<145 \mathrm{~cm})$ & Mother $<145 \mathrm{~cm}$ \\
\hline Household: Asset score (0-10) & $\begin{array}{l}\text { Asset index based on principal components analysis across the pooled three } \\
\text { rounds of data. Specifically, the first principal component of a set of } 7 \\
\text { variables (ownership of a radio, TV, bicycle, motorbike, car; household } \\
\text { electricity, improved flooring) was extracted, and re-scaled to vary between } \\
0-10 \text {. }\end{array}$ \\
\hline Household: Yrs sec. education & Sum of the maternal and paternal years of secondary education. \\
\hline Household: Improved water $(0 / 1)$ & Improved drinking water according to JMP/WHO definition \\
\hline Household: Improved toilet $(0 / 1)$ & Flush toilets or improved pit latrines \\
\hline Household: ANC blood tests $(0 / 1)$ & Mother had blood test during pregnancy \\
\hline Village: Bed net ownership (0-1) & Cluster share of households owning bed nets \\
\hline Village: Medical births $(0-1)$ & Cluster share of children born in a medical facility \\
\hline Village: Rural (0/1) & Cluster defined as rural in DHS \\
\hline Village: non-farm occupations $(0-1)$ & Share of men in a cluster reporting primary occupation in non-farm sector \\
\hline Region: ART knowledge (0-1) & $\begin{array}{l}\text { Lagged region-level share of women } 15-49 \text { years of age who know that drugs } \\
\text { can prevent mother-to-child-transmission of HIV. }\end{array}$ \\
\hline Region: HIV testing (0-1) & $\begin{array}{l}\text { Lagged region-level share of men and women } 15-49 \text { years of age who were } \\
\text { tested for HIV in the past } 12 \text { months. }\end{array}$ \\
\hline Region: Malaria prevalence 2-9 yrs & $\begin{array}{l}\text { Estimated malaria prevalence for children } 2-9 \text { years of age in the year of each } \\
\text { child's birth, averaged at the region level; used as an interaction variable. }\end{array}$ \\
\hline Region: HIV prevalence. & $\begin{array}{l}\text { Lagged region-level share of men and women } 15-49 \text { years of age estimated to } \\
\text { be HIV infected; used as an interaction variable. }\end{array}$ \\
\hline
\end{tabular}


Finally, as was the case with bednets and malaria, we expect HIV programming proxies to have a greater influence on child nutrition in areas with high initial HIV prevalence. HIV prevalence for women 15-49 months at the region level is also taken from the AIS surveys described above and used in interaction terms.

\subsection{Methods}

\subsubsection{Descriptive analysis}

After defining these variables, we then go on to analyze trends in HAZ/stunting and the various determinants of child stunting. We compute trends in survey-weighted means for all variables at the national, rural/urban, and subnational level. We then use non-parametric plots to look at changes in the distribution of HAZ, and how HAZ varies with child age, in a manner similar to the influential study by Victora et al. (2009). When looking at child growth, age-specific plots are important for examining the timing of growth faltering, so as to ascertain whether growth improvements stem from increases in birth size or from improved postnatal growth. However, because the main hypothesized mechanism linking HIV/AIDS to child growth is MTCT, we also look at population-level estimates of maternal weight by months of pregnancy to assess population-level maternal weight gain. For both population-level growth faltering in children and weight gain during pregnancy, we separately analyze patterns for low and HIV prevalence regions to explore whether there are signs of systematic differences.

\subsubsection{Regression analysis}

We use linear probability models to regress stunting $(\mathrm{N})$ for individual $i$ at time $t$ and a vector of timevarying intermediate determinants $(\mathbf{X})$, and a vector of control variables (maternal height, child and maternal age dummies, subnational fixed effects; $\boldsymbol{\mu}_{\mathbf{i}}$ ), trend effects represented by a vector of DHS survey year dummy variables $(\mathbf{T})$. The vector of coefficients $(\boldsymbol{\beta})$ represent estimated effects of these underlying determinants on nutrition outcomes constitutes the set of parameters of principal interest. With the addition of a standard white noise term $(\varepsilon)$, we represent this relationship by equation (1): 
(1) $\mathrm{N}_{\mathrm{i}, \mathrm{t}}=\boldsymbol{\beta} \mathbf{X}_{\mathrm{i}, \mathrm{k}}+\boldsymbol{\mu}_{\mathrm{i}}+\mathbf{T}+\varepsilon_{\mathrm{i}, \mathrm{t}}$

We implement equation (1) for different age ranges. This is particularly important for child growth outcomes because these indicators are cumulative in nature, and because the timing of growth faltering can change over time. For example, earlier regression-decomposition studies of Bangladesh and Nepal found that most of the stunting improvement seemed to occur at birth, suggesting the main drivers of stunting reduction were prenatal in nature. The identification of prenatal drivers of nutrition therefore require use of younger samples of children. However, for postnatal factors Alderman and Headey (2018) argue that older samples of children should be analyzed, with the expectation that the full effects of postnatal growth factors are best captured in samples of children who have completed the first 1000 days of life (i.e. 24 months and older). For example, whilst children exclusively breastfed (e.g. 0-5 months) will be largely protected from poor water quality, a child 24 months of age may have been exposed to poor water for a large portion of its vulnerable first 1000 days of life, and hence may have incurred a sizeable growth penalty.

Because of these issues, we stratify our regressions into the following sub-samples:

1. $0-59$ months (all available ages);

2. $0-5$ months (to best capture prenatal and neonatal factors);

3. 6-23 months (to capture growth faltering in a period of high nutrient requirements and increasing vulnerability to infection); and

4. 24-59 months (to capture the full impacts of postnatal growth factors, such as household wealth, education, and WASH status). 


\subsubsection{Decomposition analysis}

While equation (1) says something about the importance of underlying determinants in explaining crosssectional variation in nutrition outcomes, the regression approach by itself does not inform the question of which factors have been driving nutritional changes over time in Tanzania. For this a decomposition of the regression model is required. The simplest decomposition of stunting change over 2005-2016 assumes that the effects of $\mathbf{X}$ on nutrition outcomes is unchanged over time (e.g., maternal education is just as effective in 2005 as it is in 2015-16), which can be tested. If these tests reveal no strong evidence of coefficient changes over time, then the contribution of each explanatory variable to nutritional change over time is the product of its coefficient times the change in its mean value over time:

(2) $\Delta \mathrm{N}_{\mathrm{i}, \mathrm{t}}=\boldsymbol{\beta} \Delta \mathbf{X}_{\mathrm{i}, \mathrm{k}}$

In addition to retrospectively exploring what might have been driving nutritional change in Tanzania, regression models can also be used for predictive analysis. The key assumption here is that the effects of each underlying determinant on nutrition $(\mathbf{X})$ are unchanged over time. Then, instead of comparing predicted change in the past to the actual retrospective change, one can make comparisons between predicted change in the future to the targeted change in the future (such as the WHA 2025 stunting reduction target). This analysis gives planners a sense of the types of factors that need to be accelerated to achieve rapid stunting reduction in the near future.

\subsubsection{Projection analysis}

Equation (2) can be used to examine historical changes in $\mathbf{X}$, but it can also be used to test the potential stunting changes resulting from future projected/assumed changes in $\mathbf{X}$ variables. We therefore use the regression coefficients from 1 in conjunction with assumed changes in $\mathrm{X}$ to examine what types of changes in nutrition-specific and nutrition-sensitive factors would be required to achieve the WHA target of a $40 \%$ reduction in stunting by 2025 . 


\section{DESCRIPTIVE RESULTS}

In this section we report a series of descriptive results to understand patterns and trends of stunting in Tanzania, and changes in some of the potential drivers of stunting changes.

\subsection{Trends in stunting}

Table 3.1 reports trends in child stunting rates for Tanzania as a whole, as well as rural and urban areas, and the nine ecological zones. Progress in rural and urban areas was quite similar, with both experiencing declines of around 9 percentage points. In most regions the bulk of the reduction in stunting occurred over the 2010-2016 period, although the Southern, Southern Highlands, and Western regions also saw significant reductions over 2005-2010. These regions were also the best-performing regions over the entire 10-year period, recording very rapid stunting reductions of 21.7 points (Southern), 15.6 points (Southern Highlands) and 17.2 points (Western). Also notable is that the Southern and Southern Highland zones had very high stunting rates to begin with (almost $60 \%$ of children were stunted in 2005). Indeed, the patterns over all zones show strong signs of spatial convergence over time, or "catch-up". Specifically, the correlation between changes in stunting over 2005-2016 and initial stunting rates in 2005 is a very high -0.75 . However, despite this convergence in stunting rates across regions, significant disparities still persist. Stunting rates in the Southern Highlands and South-Western Highlands in 2015-16 were at least 5 percentage points higher than the next highest regional stunting rate, and urban areas have stunting rates some 12.3 percentage points smaller than rural rates. 
Table 3.1 Trends in stunting rates for nine ecological zones, rural and urban areas, and all Tanzania (children 0-59 months of age)

\begin{tabular}{lcccc}
\hline & $\mathbf{2 0 0 5}$ & $\mathbf{2 0 1 0}$ & $\mathbf{2 0 1 5 - 1 6}$ & Change \\
\hline Lake & $41.0 \%$ & $39.9 \%$ & $35.5 \%$ & $-5.5 \%$ \\
Western & $49.5 \%$ & $39.4 \%$ & $32.2 \%$ & $-17.2 \%$ \\
Northern & $38.7 \%$ & $41.8 \%$ & $37.3 \%$ & $-1.4 \%$ \\
Central & $48.1 \%$ & $49.1 \%$ & $32.8 \%$ & $-15.3 \%$ \\
South-Western Highlands & $50.1 \%$ & $51.8 \%$ & $42.5 \%$ & $-7.6 \%$ \\
Eastern & $32.5 \%$ & $32.1 \%$ & $23.3 \%$ & $-9.3 \%$ \\
Southern Highlands & $60.3 \%$ & $48.2 \%$ & $44.7 \%$ & $-15.6 \%$ \\
Southern & $57.6 \%$ & $44.9 \%$ & $35.8 \%$ & $-21.7 \%$ \\
Zanzibar & $27.2 \%$ & $29.4 \%$ & $23.6 \%$ & $-3.6 \%$ \\
& & & & \\
Rural Tanzania & $46.8 \%$ & $44.5 \%$ & $37.4 \%$ & $-9.4 \%$ \\
Urban Tanzania & $33.8 \%$ & $31.2 \%$ & $25.1 \%$ & $-8.7 \%$ \\
All Tanzania & & & & $-10.1 \%$ \\
\hline
\end{tabular}

Source: Authors' calculations from Tanzania DHS.

While stunting is conventionally reported for all children 0-59 months, it is also well known that most stunting occurs prior to a child's second birthday (Victora et al., 2009), either in utero (reflected in small birth size), infancy, or early childhood, when a child has high nutrient requirements and elevated vulnerability to infection. For this reason the relationship between HAZ/stunting and child age is especially informative. This is shown in Figure 3.1, where the curves are based on flexible non-linear regression smoothing to plot stunting rates as a function of age (which is also measured with some error), along with $90 \%$ confidence intervals. In each figure the solid line represents $2004-05$ while the longdashed line represents 2010, and the short-dashed represents 2015-16.

In Panel A, which shows predicted HAZ, we observe a significant intercept shift, signalling an improvement in birth size. The results in Panel B, which shows the predicted prevalence of stunting, suggest neonatal stunting is at least 5 percentages points lower in 2015-16, although the small sample of young children in the DHS makes the estimate imprecise. However, the stunting curve in Panel B suggests that there is also postnatal improvement in growth faltering, with the gap between the 2004-05 
and 2015-16 curves widening between 6 and 24 months. In summary, improvements in linear gorwth in Tanzania stem from a combination of birth size improvements and postnatal growth improvements.

The result in Figure 3.1 are informative, but Tanzania is geographically diverse, with large rural-urban differences in stunting, and substantial variation in factors such as malaria and HIV prevalence. In Figure 3.2 we examine this diversity by examining rural and urban stunting patterns by child age. In Panel A, which depicts stunting in rural areas, the plots broadly resemble those for the country as a whole, with some suggestion of an increased mean birth size, but also some improvement in postnatal growth faltering. In urban areas the pattern is quite different, however, with no strong suggestion of a birth size improvement (although the smaller urban sample reduces precision), but a substantial reduction in stunting in the 6-24 month period. 
Figure 3.1 HAZ and stunting as a regression-based function of child age, 2005 and 2015-16

\section{Panel A: Predicted HAZ and child age}

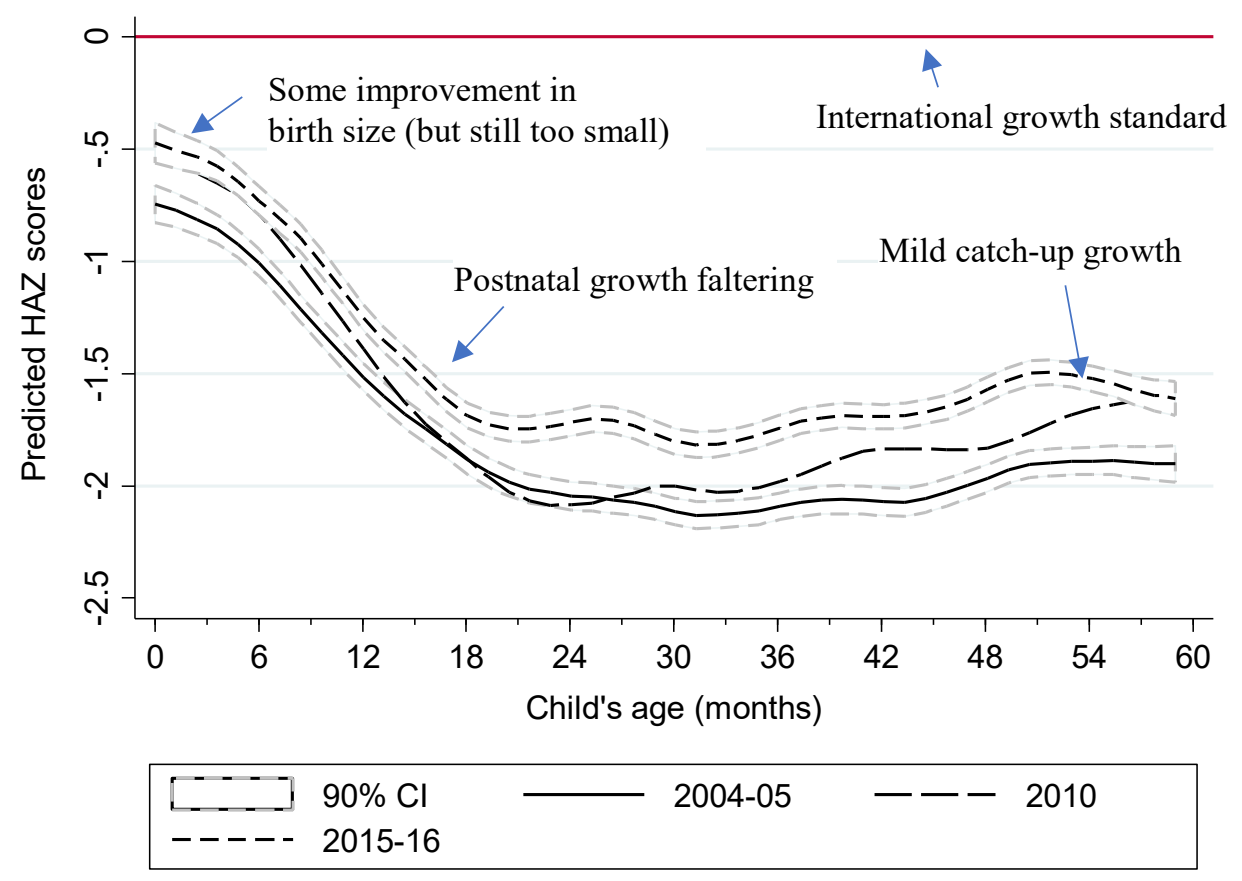

\section{Panel B: Predicted stunting prevalence and child age}

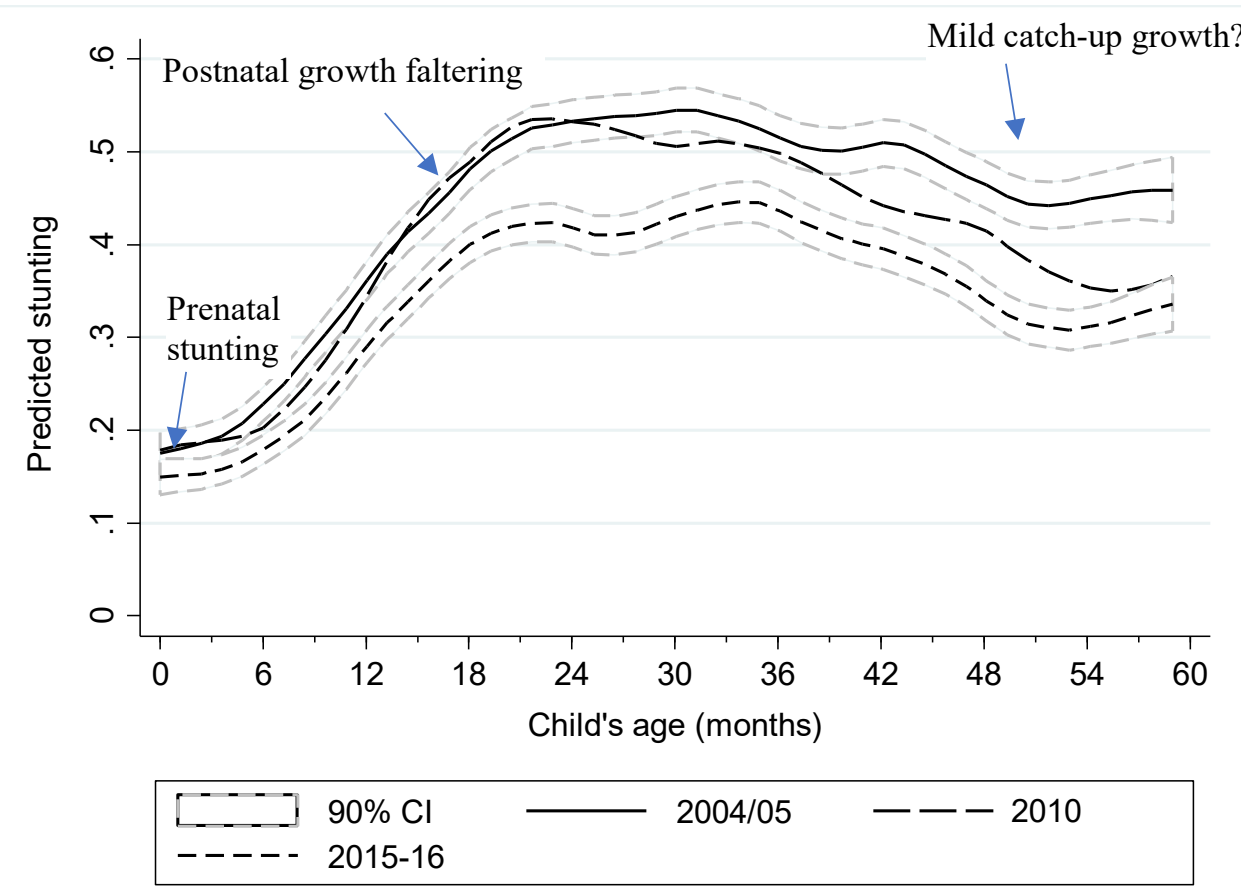

Source: Authors' calculations from Tanzania DHS using the LPOLY regression in STATA (lpolyci command). 
Figure 3.2 Stunting and child age in rural and urban areas, 2004-05 and 2015-16

\section{Panel A: Rural Areas}

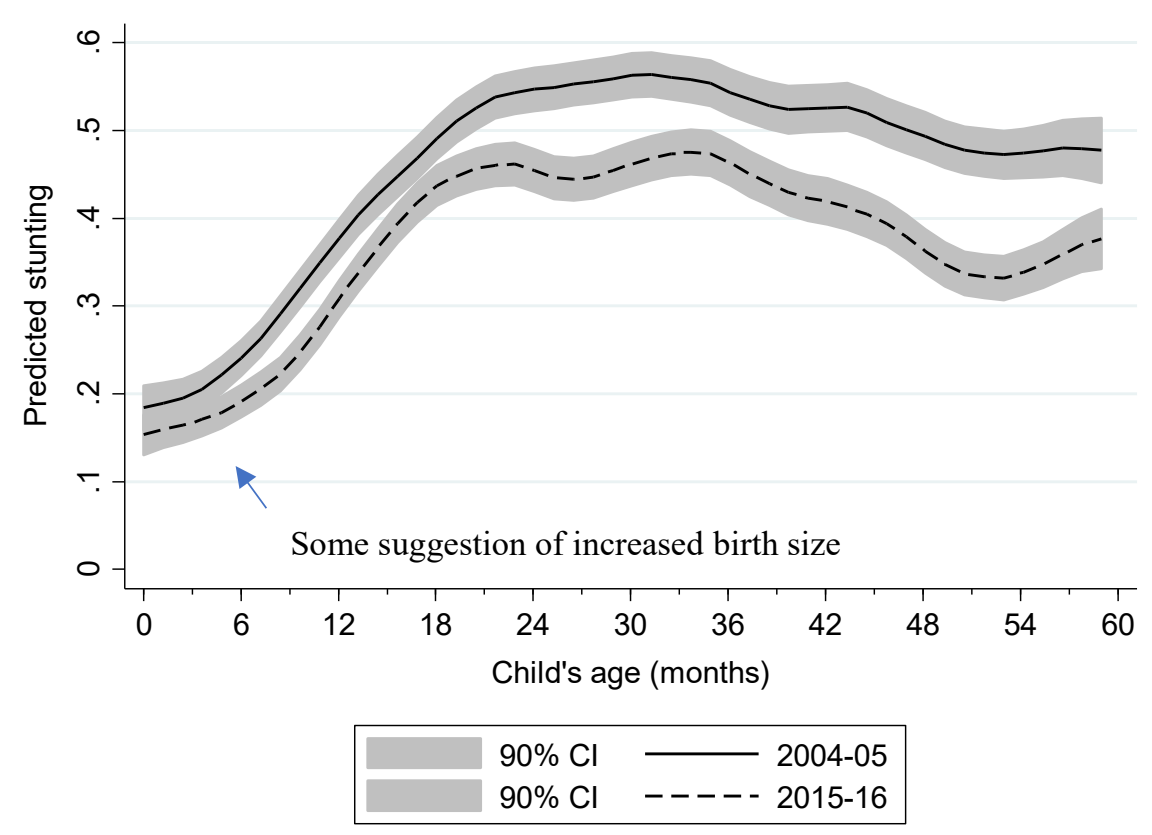

\section{Panel B: Urban areas}

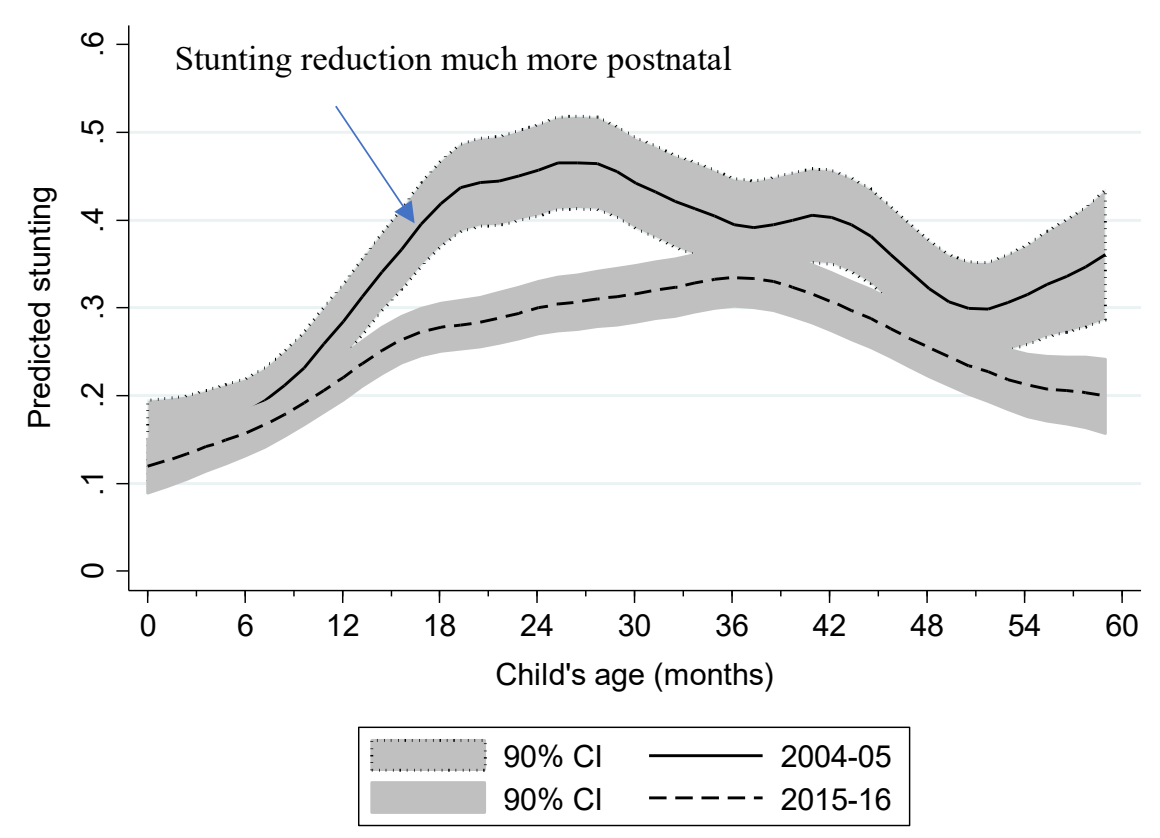

Source: Authors' calculations from Tanzania DHS using the LPOLY regression in STATA (lpolyci command). 
In Figure 3.3 we examine stunting-age relationships in low and high HIV areas, since mother-to-childtransmission ought to affect birth size, and improved MTCT prevention should result in improvements in birth size. In Panel A (low HIV regions where HIV prevalence<5\%) improvements in growth are characterized by a reduction in postnatal stunting, particularly from around 9 months onwards. In contrast, stunting reduction in high HIV areas (HIV prevalence $>7.5 \%$ ) is very much characterized by improvements in birth size (Panel B).

In Figure 3.4 we further explore the issue of birth size improvements by looking at maternal weight during pregnancy for low and high HIV areas. The y-axis denotes months of pregnancy as reported by the mother herself (most likely with some error). ${ }^{2}$ We use $90 \%$ confidence intervals to engage in a one-sided test of the null hypothesis that maternal weight gain during pregnancy is significantly greater in 2015-16 compared to earlier rounds. The result shows a large and statically significant improvement in maternal BMI during pregnancy between the two earlier rounds and the 2015-16 round. Maternal BMI becomes significantly larger in the 2015-16 round at the start of the second trimester - precisely when weight gain becomes important for appropriate fetal development - and then further improves over the course of the second trimester. Indeed, the difference at 6 months gestation approximately 5 kilograms. For most of the last trimester maternal BMI continues to be much higher, although at 9 months the difference is no longer significant, presumably because there are important selection issues that induce measurement error (e.g., pre-term births, or errors in gestation estimates). All in all, the results in Figure 3.4 are strongly consistent with the marked reduction in neonatal stunting observed in high HIV areas.

\footnotetext{
${ }^{2}$ We pool the 2004-05 and 2010 DHS rounds together because there was no change in neonatal stunting observed over the 200405 and 2010 round; (b) because we also observed no difference in weight patterns when we plot Figure 3.6 separately for the two earlier rounds; and (c) pooling the two rounds improves the precision of the plot below, since sample sizes for pregnant women are relatively small in each individual round (approximately 1000 women per round).
} 
Figure 3.3 Stunting by age in low and high HIV prevalence regions, 2010 and 2015-16

Panel A: Lower HIV areas (prevalence among adult women lower than 5\%)

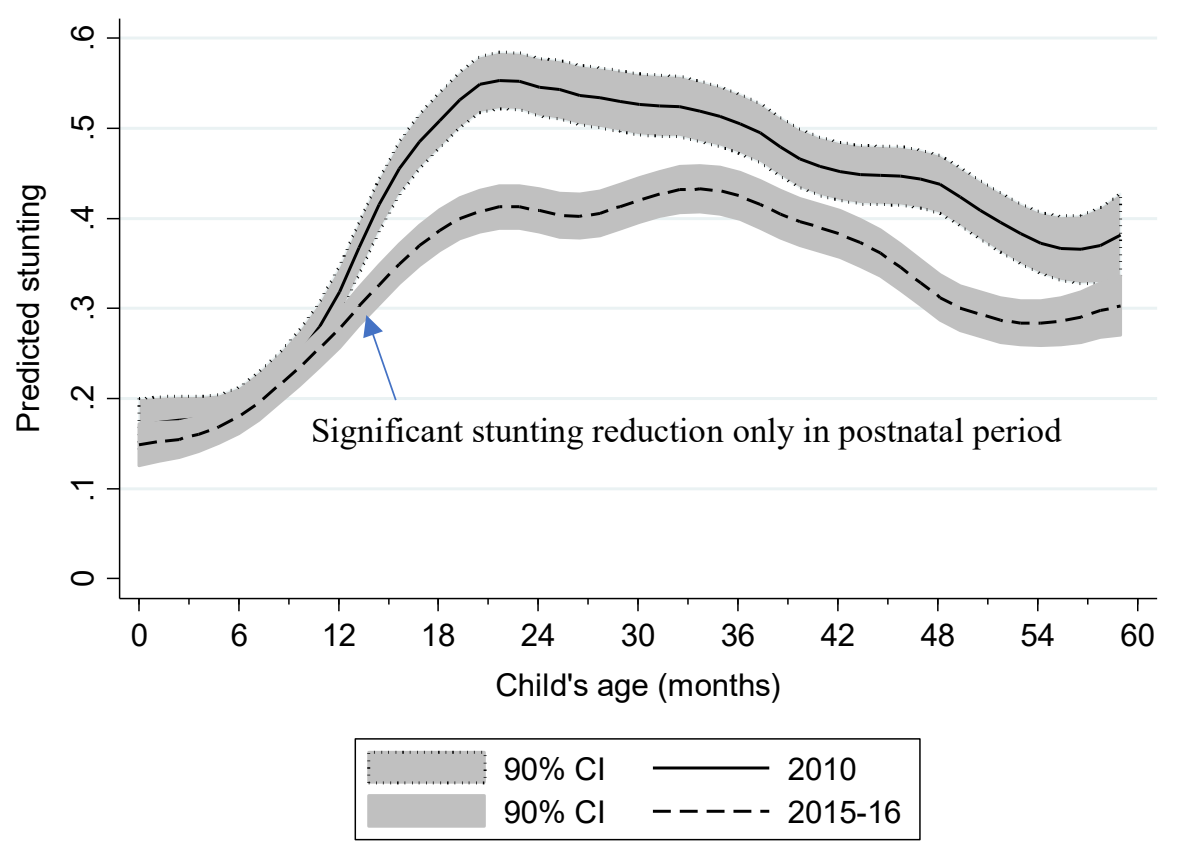

\section{Panel B: High HIV areas (prevalence among adult women greater than $7.5 \%$ )}

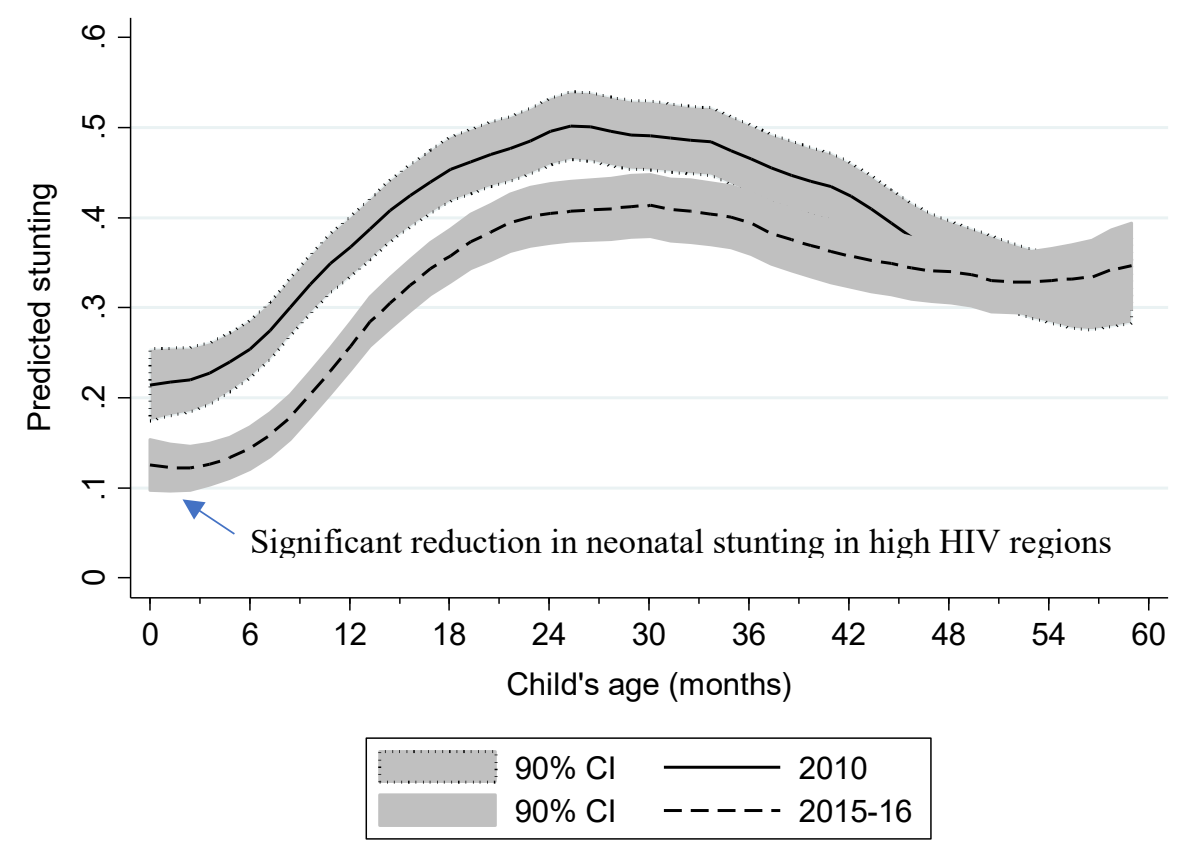

Source: Authors' calculations from Tanzania DHS using the LPOLY regression in STATA (lpolyci command). 
Figure 3.4 Changes in maternal weight (kg) by month of pregnancy between 2015-16 and the 200405 and 2010 DHS rounds, for low and high HIV prevalence regions

\section{Panel A: Low HIV areas (prevalence among adult women lower than 5\%)}

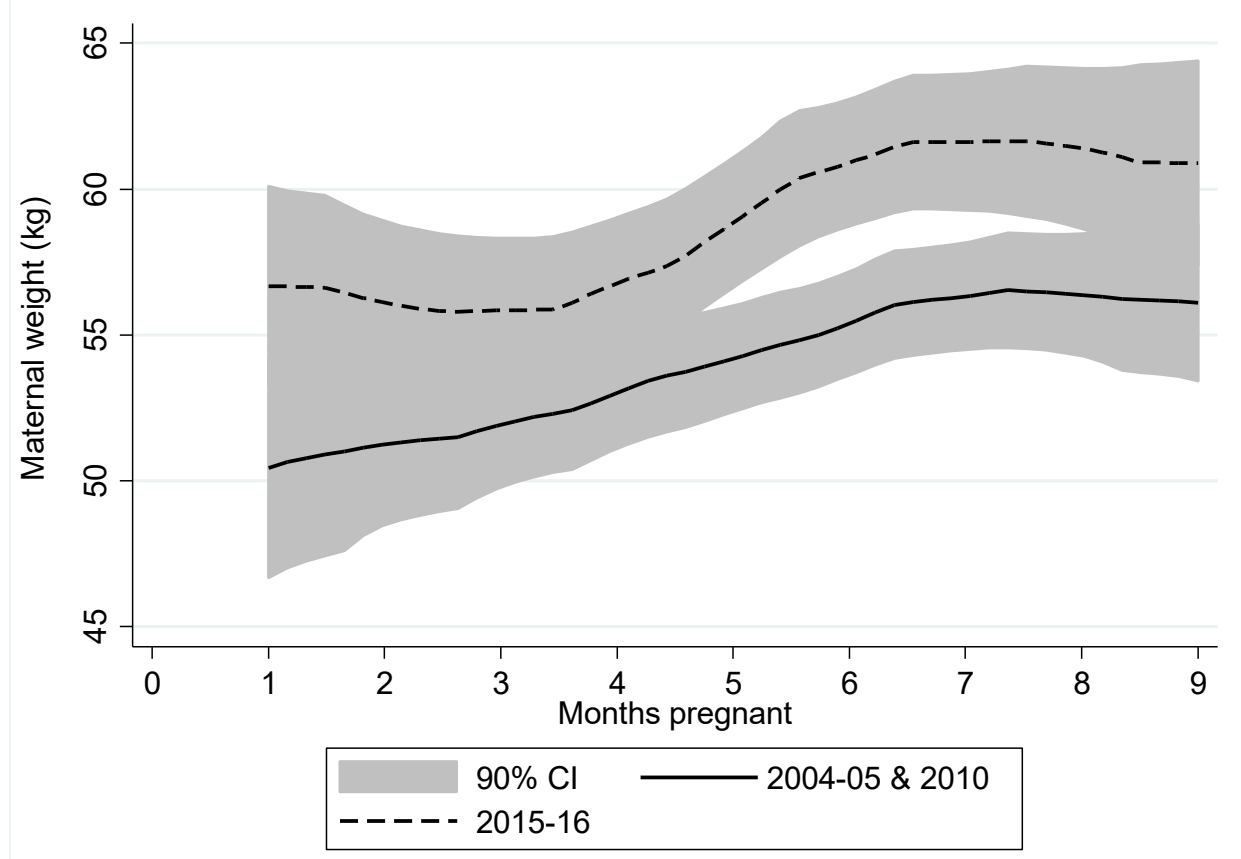

Panel B: High HIV areas (prevalence among adult women greater than $7.5 \%$ )

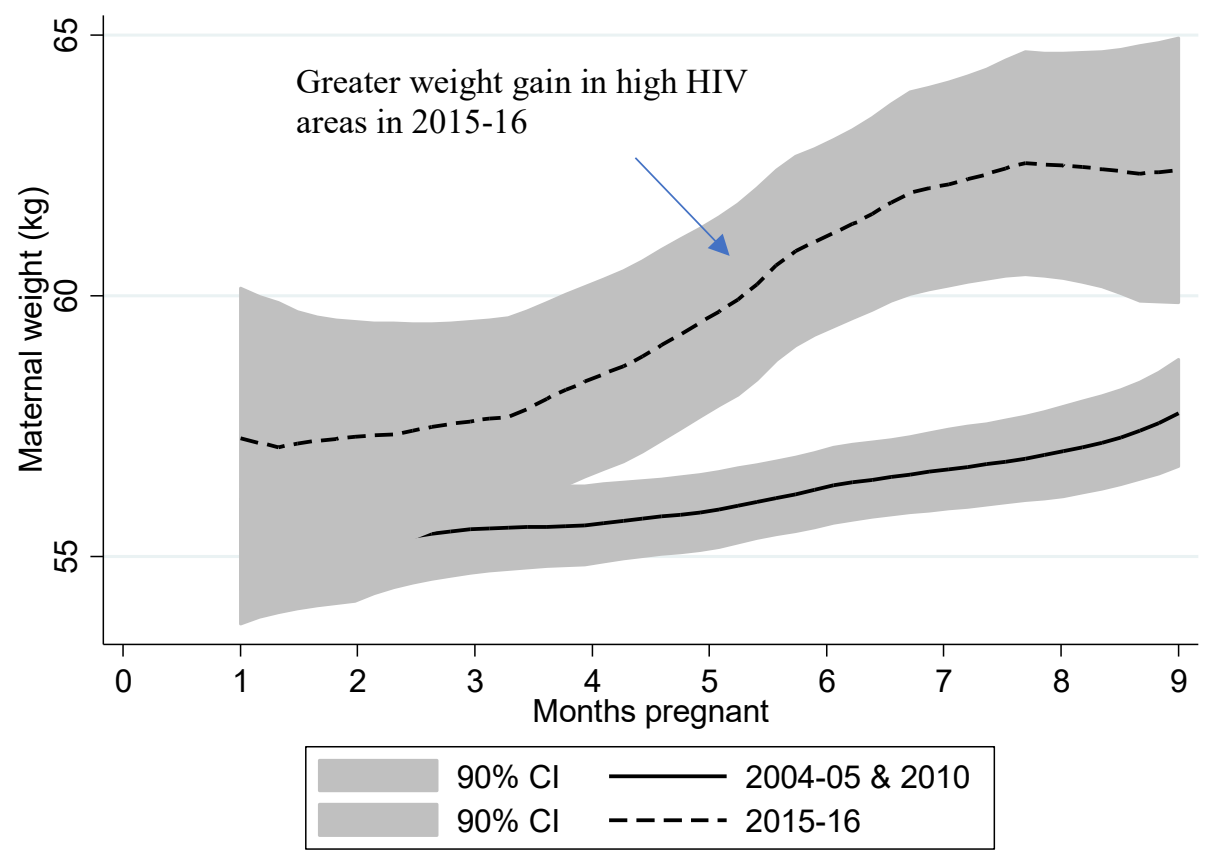

Source: Authors' calculations from Tanzania DHS using the LPOLY regression in STATA (lpolyci command). Samples sizes are 1,056 for the 2004-05 round, 926 for the 2010 round and 996 for the 2015-16 round. 


\subsection{Trends in potential determinants of stunting}

What about changes in other predictors of dietary or health improvements? Table 3.2 reports changes in various underlying determinants of stunting over 2005. There are some substantial changes across a wide range of indicators. The asset index (0-10), which is usually very strongly associated with stunting reductions, increased from just 0.96 out of 10 to 1.57 out of 10 . In other words, the asset score improved by about $60 \%$, but from a very low base such that most Tanzanians in 2015-16 remained asset-poor.

Likewise, we see only a relatively modest improvement in education: an additional 1.18 years of secondary education for both parents in a household. These moderate socioeconomic improvements suggest that dietary improvements are perhaps unlikely to be the main driver of increases in birth size or postnatal growth; indeed, we show below that there is no evidence that children's dietary diversity improved.

Table 3.2 Changes in various underlying determinants of stunting, 2005-2016

\begin{tabular}{|c|c|c|c|c|c|}
\hline & 2005 & 2010 & 2015-2016 & $\begin{array}{l}\text { Change } \\
\text { 2005-16 }\end{array}$ & $\begin{array}{c}\text { Percentage } \\
\text { change } \\
2015-16\end{array}$ \\
\hline Female child $(0 / 1)$ & 0.50 & 0.50 & 0.49 & -0.01 & $-2 \%$ \\
\hline Birth interval $<18 \mathrm{~m}(0 / 1)$ & 0.12 & 0.12 & 0.14 & 0.02 & $17 \%$ \\
\hline Adolescent mother $(0 / 1)$ & 0.13 & 0.13 & 0.14 & 0.01 & $8 \%$ \\
\hline Low BMI $(0 / 1)$ & 0.07 & 0.09 & 0.07 & 0.00 & $0 \%$ \\
\hline Mother short $(0 / 1)$ & 0.02 & 0.03 & 0.02 & -0.01 & $0 \%$ \\
\hline Asset index $(0-10)$ & 0.96 & 1.19 & 1.57 & 0.62 & $64 \%$ \\
\hline Years of secondary education & 1.79 & 1.78 & 2.96 & 1.18 & $65 \%$ \\
\hline Improved water $(0 / 1)$ & 0.44 & 0.45 & 0.57 & 0.12 & $30 \%$ \\
\hline Improved toilet $(0 / 1)$ & 0.04 & 0.16 & 0.28 & 0.23 & $600 \%$ \\
\hline Antenatal blood tests $(0 / 1)$ & 0.35 & 0.51 & 0.60 & 0.25 & $71 \%$ \\
\hline Household owns bed net $(0 / 1)$ & 0.50 & 0.89 & 0.78 & 0.28 & $56 \%$ \\
\hline Village medical births $(0-1)$ & 0.47 & 0.50 & 0.63 & 0.16 & $34 \%$ \\
\hline non-farm occupations $(0-1)$ & 0.28 & 0.35 & 0.49 & 0.21 & $75 \%$ \\
\hline Region: ART knowledge (0-1) & 0.14 & 0.51 & 0.69 & 0.55 & $393 \%$ \\
\hline
\end{tabular}

Source: Authors' calculations from Tanzania DHS. 
What about potential drivers of infection? In terms of WASH indicators, we observe a 12 percentage point improvement in access to improved water, mostly over the $2010-2016$ period, and a 23 percentage points improvement in access to an improved toilet, from an extremely low base (just $4 \%$ in 2005). Access to antenatal and neonatal health services has improved. The share of births taking place in a medical facility increased from $47 \%$ to $63 \%$, with most of the increase over $2010-2016$, while antenatal blood tests increased from $35 \%$ in 2005 to $60 \%$ in $2015-16$.

Another significant change is in malaria prevention and treatment. Here we capture malaria prevention through ownership of bednets, which increased from 50\% in 2005 to $89 \%$ in 2010, before falling back to $78 \%$ in $2015 / 16$. The DHS also records Intermittent Preventive Treatment of malaria in pregnant women by asking whether FANSIDAR was taken during pregnancy. This indicator increased from $44.5 \%$ of mothers in 2005 to $64.8 \%$ in 2010 and $70.6 \%$ in $2015-16$. In addition, estimates of malaria prevalence among children 2-9 years of age from the Malaria Atlas Project is reported in Figure 3.5 to show the spatial pattern of changes in malaria, while the table in Figure 3.5 reports malaria prevalence at the time of a child's birth for all children surveyed in 2005 .

In the 2005 DHS these neonatal malaria rates were highest in the Southern Zone where some $54.9 \%$ of children 2-9 years of age were estimated to be infected, but also relatively high in Zanzibar and the Western, Eastern and Lake zones ( 20\%). Only the Southern Highland and Central zones had malaria prevalence below $10 \%$. Remarkably, by 2015 , malaria prevalence was estimated to be less than $5 \%$ in all zones. The fact that malaria prevalence in 2005 was highest in the Southern zone - a zone which experienced the most rapid reduction in stunting - suggests that efforts to address malaria in Tanzania may have had significant impacts on child stunting. 
Figure 3.5 Changes in malaria prevalence among children 2-9 years of age
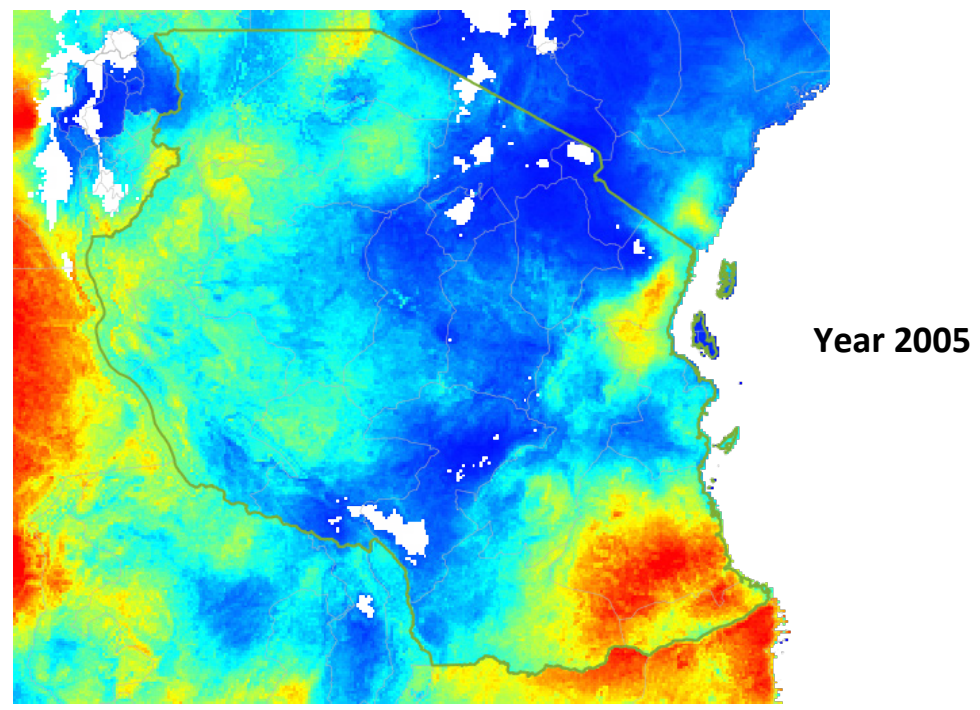

\begin{tabular}{|c|c|}
\hline Zones & Prevalence \\
\hline Lake & $16.7 \%$ \\
\hline Western & $19.8 \%$ \\
\hline Northern & $14.1 \%$ \\
\hline Central & $5.5 \%$ \\
\hline South West Highlands & $9.6 \%$ \\
\hline Eastern & $22.0 \%$ \\
\hline Southern Highlands & $9.7 \%$ \\
\hline Southern & $54.9 \%$ \\
\hline Zanzibar & $22.8 \%$ \\
\hline
\end{tabular}
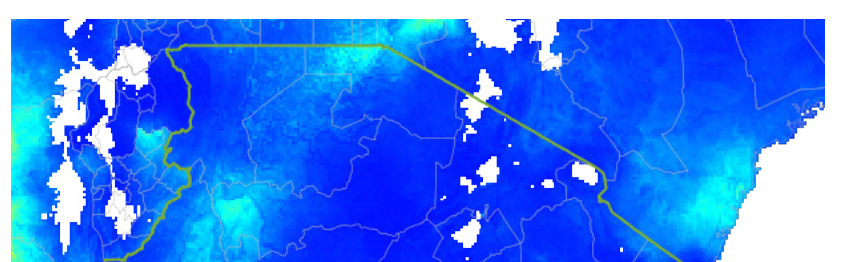

Legend (malaria prevalence)

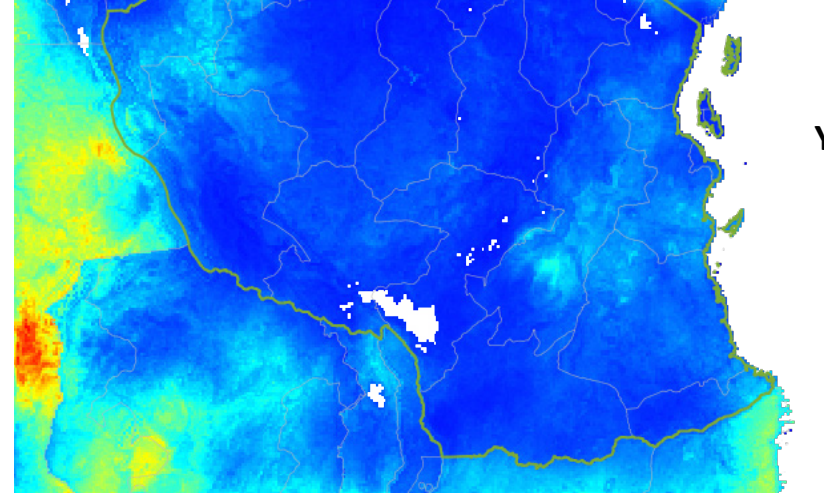

Year 2015

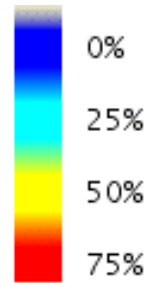

Source: Bhatt et al. (2015).

In addition to combating malaria, the mid 2000s saw signifiant efforts aimed at preventing and treating HIV/AIDS, including efforst to prevent mother-to-child transmission (MTCT). Geographically, HIV prevalence among adults is highest in the Southern Highlands (Figure 3.6), but also relatively high in the more urbanized eastern regions and Zanzibar, as well as the Lake regions. Unlike other disease indicators, for which urban populations are almost invariably better off, HIV prevalence tends to be higher in urban 
areas (on average, urban HIV prevalence $(\sim 10 \%)$ is double the rate of rural areas, $\sim 5 \%$ ), and also higher among somewhat more educated and wealthier adults.

Figure 3.6 A map of HIV prevalence among women 15-49 years of age, 2007

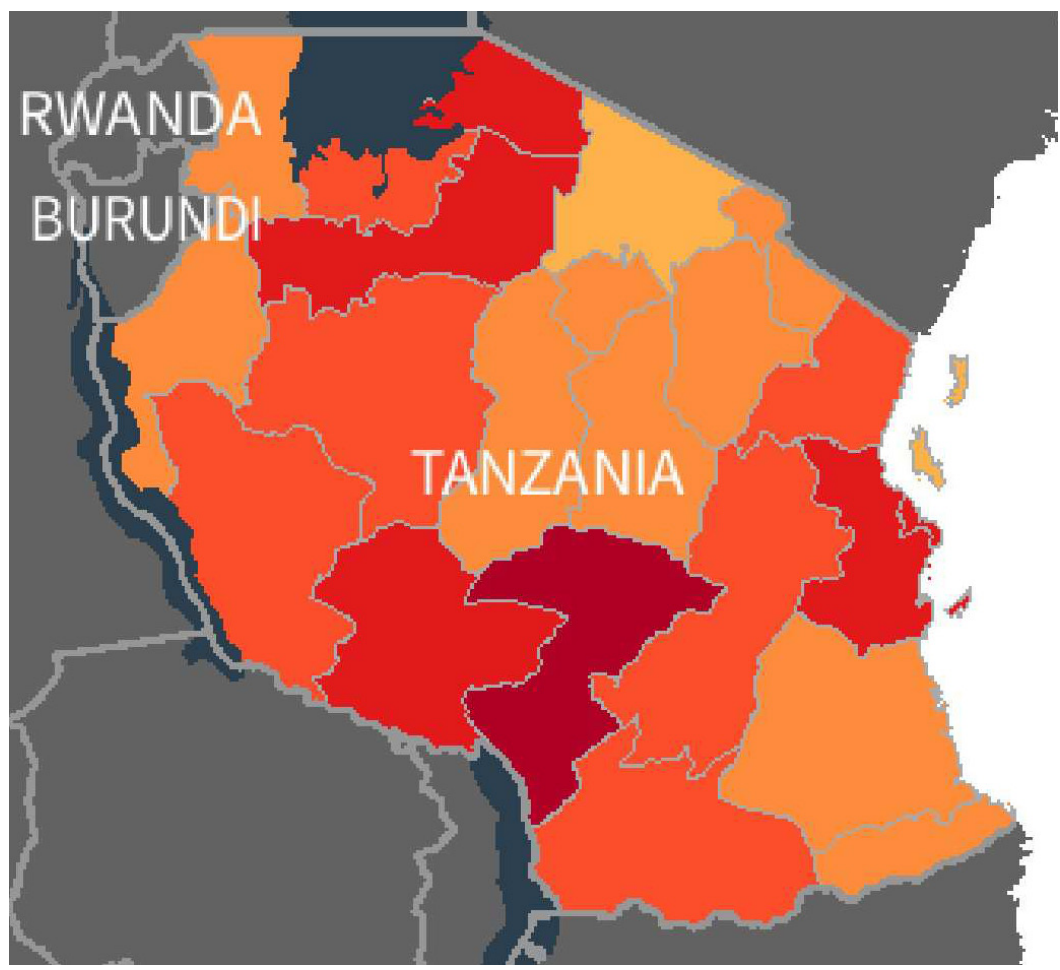

\begin{tabular}{|l|l|}
\hline Legend & \\
\hline Up to 0.9 & \\
\hline 1.0 to 4.9 & \\
\hline 5.0 to 7.4 & \\
\hline 7.5 to 11.3 & \\
\hline 11.4 and higher & \\
\hline
\end{tabular}

Source: Map produced from DHS STATCOMPILER data (ICF-International \& USAID, 2017).

AIS data suggest that HIV testing in the past 12 months among adults increased rapidly over time, from just $5 \%$ in 2003 , to $21 \%$ in 2008 and $28 \%$ in 2012 (Table 3.3). The 2007-08 and 2011-12 AIS also antenatal HIV testing and counseling, as all three rounds of AIS record various measures of HIV/AIDS knowledge. Interestingly, basic HIV and AIDS knowledge improved very little over time, but PMTCT knowledge through antiretroviral therapy (ART) improved dramatically, increasing from $14.7 \%$ in 2003/04 to $64.3 \%$ in 2011-12. HIV testing and counseling also increased substantially over 2007-08 to 2011-12, from $43.3 \%$ to $76.7 \%$. For both indicators, improvements took place in rural and urban areas, although HIV testing and counseling increased much more rapidly in rural areas, albeit from a lower base. 
Table 3.3 Trends in HIV/AIDS knowledge, and antenatal testing and counseling

\begin{tabular}{|c|c|c|c|}
\hline & HIV/AIDS knowledge ${ }^{\mathrm{a}}$ & ART knowledge ${ }^{b}$ & $\begin{array}{c}\text { Antenatal HIV testing } \& \\
\text { counseling }\end{array}$ \\
\hline \multicolumn{4}{|l|}{ All Tanzania } \\
\hline 2003-04 AIS & $36.8 \%$ & $14.7 \%$ & \\
\hline 2007-08 AIS & $39.4 \%$ & $49.3 \%$ & $43.3 \%$ \\
\hline 2011-12 AIS & $42.0 \%$ & $64.3 \%$ & $76.7 \%$ \\
\hline Change & 5.2 points & 49.6 points & 23.4 points \\
\hline \multicolumn{4}{|l|}{$\underline{\text { Rural areas }}$} \\
\hline 2003-04 AIS & $32.5 \%$ & $9.7 \%$ & \\
\hline 2007-08 AIS & $34.8 \%$ & $43.3 \%$ & $37.0 \%$ \\
\hline 2011-12 AIS & $37.3 \%$ & $59.3 \%$ & $73.7 \%$ \\
\hline Change & 4.8 points & 51.4 points & 35.3 points \\
\hline \multicolumn{4}{|l|}{$\underline{\text { Urban areas }}$} \\
\hline 2003-04 AIS & $46.3 \%$ & $25.8 \%$ & \\
\hline 2007-08 AIS & $52.4 \%$ & $66.1 \%$ & $73.5 \%$ \\
\hline 2011-12 AIS & $55.0 \%$ & $78.0 \%$ & $90.3 \%$ \\
\hline Change & 8.7 points & 72.2 points & 16.8 points \\
\hline
\end{tabular}

Notes:

a. Percentage of women who correctly identify the two major ways of preventing the sexual transmission of HIV (using condoms and limiting sex to one faithful, uninfected partner), who reject the two most common local misconceptions about HIV transmission, and who know that a healthy-looking person can have HIV.

b. Percentage of women who report that maternal to child transmission of HIV can be prevented through antiretroviral therapy during pregnancy and avoiding breastfeeding.

c. Percentage of women who were tested for HIV, received the result and received post-test counselling during antenatal visit for the most recent birth, of all women who gave birth in the two years preceding the survey.

Finally, Table 3.4 summarizes major changes within zones over 2005-16, with zones ranked by their performance in reducing stunting. Strikingly, the best performing Southern and Western regions saw major reductions in stunting (21.7 and 17.2 percentage points) accompanied by huge increases in ART knowledge (70 and 63 percentage points), antenatal blood tests ( 40 and 47 percentage points respectively), bed net ownership (39 and 62 percentage points) and moderate increases in access to improved water (19 and 15 percentage points). The Southern Highlands also saw an impressive 15.6 percentage point reduction in stunting. It, too, experienced improvements in ART knowledge (70 percentage points) and blood tests, but also saw a 33 percentage point improvement in toilet use. The similarly impressive reduction in the Central region was accompanied by a huge increase in antenatal 
blood tests (52 points). The Eastern and Zanzibar regions stand out for large increases in toilet use ( $>50$ points).

Table 3.4 Zonal changes in the various underlying determinants of stunting

\begin{tabular}{|c|c|c|c|}
\hline & $\begin{array}{l}\text { Zones } \\
\text { nked by stunting } \\
\text { change) } \\
\end{array}$ & $\begin{array}{c}\text { Change in } \\
\text { stunting }\end{array}$ & Major changes in determinants over 2005-2016 \\
\hline 1 & Southern & -21.7 points & $\begin{array}{c}\text { Medical facilities ( } 41 \% \text { points), antenatal blood tests }(40 \% \text { points }) \text {, bed nets ( } 39 \% \text { points), } \\
\text { ART knowledge ( } 70 \% \text { points), water ( } 19 \% \text { points) }\end{array}$ \\
\hline 2 & Western & -17.2 points & $\begin{array}{c}\text { Bed nets ( } 62 \% \text { points), antenatal blood tests ( } 47 \% \text { points), ART knowledge ( } 63 \% \text { points), } \\
\text { water ( } 15 \% \text { points })\end{array}$ \\
\hline 3 & $\begin{array}{l}\text { Southern } \\
\text { Highlands }\end{array}$ & -15.6 points & $\begin{array}{c}\text { Toilets (33\% points), ART knowledge ( } 70 \% \text { points), antenatal blood tests ( } 26 \% \text { points), } \\
\text { medical facilities ( } 12 \% \text { points), bed nets ( } 18 \% \text { points) }\end{array}$ \\
\hline 4 & Central & -15.3 points & $\begin{array}{l}\text { Antenatal blood tests ( } 52 \% \text { points), ART knowledge ( } 44 \% \text { points), bed nets ( } 18 \% \text { points), } \\
\text { medical facility ( } 16 \% \text { points })\end{array}$ \\
\hline 5 & Eastern & -9.3 points & $\begin{array}{c}\text { Toilets ( } 56 \% \text { points), medical facilities ( } 29 \% \text { points), ART knowledge ( } 51 \% \text { points), bed } \\
\text { nets ( } 20 \% \text { points), antenatal blood tests ( } 16 \% \text { points })\end{array}$ \\
\hline 6 & $\begin{array}{l}\text { South West } \\
\text { Highlands }\end{array}$ & -7.6 points & $\begin{array}{l}\text { Bed nets (43\% points), antenatal blood tests ( } 40 \% \text { points), ART knowledge ( } 49 \% \text { points), } \\
\text { medical facility ( } 17 \% \text { points), toilets ( } 15 \% \text { points })\end{array}$ \\
\hline 7 & Lake & -5.5 points & $\begin{array}{l}\text { Bed nets ( } 57 \% \text { points), antenatal blood tests ( } 36 \% \text { points), ART knowledge ( } 58 \% \text { points), } \\
\text { water ( } 14 \% \text { points), flesh foods/eggs ( } 8 \% \text { points })\end{array}$ \\
\hline 8 & Zanzibar & -3.6 points & $\begin{array}{l}\text { Toilets ( } 58 \% \text { points), antenatal blood tests ( } 42 \% \text { points), bed nets ( } 40 \% \text { points), medical } \\
\text { facilities ( } 19 \% \text { points), ART knowledge ( } 58 \% \text { points }) \text {, maternal education }(15 \% \text { points })\end{array}$ \\
\hline 9 & Northern & -1.4 points & $\begin{array}{l}\text { Water ( } 38 \% \text { points), toilets ( } 34 \% \text { points), antenatal blood tests }(26 \% \text { points), bed nets } \\
\text { ( } 22 \% \text { points), ART knowledge ( } 38 \% \text { points })\end{array}$ \\
\hline
\end{tabular}

Source: Authors' estimates from DHS and AIS data. 


\section{REGRESSION ANALYSIS}

\subsection{Main regression results}

Table 3.5 reports results from a linear probability model with stunting as a function of the variables described above. All regressions control for 26 regional fixed effects, survey dummies for 2010 and 2015, as well as age-in-month dummies to reflect generic growth faltering patterns. As was also noted above, we conduct additional analysis in which we split the full sample of children 0-59 months into various age groups, because each sample has different advantages and disadvantages in terms of analyzing different determinants of stunting.

To begin with, we focus on results for all children 0-59 months in Column (1). These suggest that girls are 5 points less likely to be stunted (a standard result), and children born after a short birth interval or to an adolescent mother are more likely to be stunted. Maternal BMI is negatively associated with stunting, and short mothers (of which there are relatively few in Tanzania) are 25 points more likely to be stunted. The household asset score (varying from 0-10) is negatively associated with stunting: each 1-point movement decreases stunting by 1.8 points. Years of parental secondary education also share a negative association, but a relatively weak one. Improved water and sanitation are modestly but significantly associated with stunting in the 0-59 month range, but ANC blood tests are not significantly associated with stunting, except in the 6-23 month range. However, village-level medical births has a similarly strong association with stunting, while non-farm employment in a village has a marginally significant association.

The last four indicators refer to malaria and HIV-related factors. Here we allow for the effects of bednets and ART knowledge to be larger in high malaria or high HIV regions. In low malaria regions, for example, moving from zero bednet use to $100 \%$ bednet use predicts that a child is 8.2 points less likely to be stunted. However, if the child is located in a high malaria region, then the effect increases by 4.2 points, and the child is 12.4 points less likely to be stunted. In low HIV regions we find no significant 
impact of ART knowledge, whereas in high HIV areas moving from $0 \%$ to $100 \%$ ART knowledge predicts a 7.3 point reduction in stunting risk.

In the remaining regressions we see some expected patterns, based on previous research on age sensitivity in stunting associations (Alderman and Headey 2018). Factors that tend to have most of their impact in early childhood, rather than prenatally, tend to see stronger associations in the 24-59 month range, including assets, education, water and sanitation. Bednet use also remains significant in this period, but ART knowledge is no longer significant in the 24-59 month sample. However, when we move to younger samples ART knowledge becomes significant in high HIV prevalence regions, and indeed the coefficient increases in magnitude to -0.102 in the $6-24$ month sample and -0.131 in the $0-5$ month sample. This pattern of ART knowledge results is consistent with MTCT primarily affecting children through prenatal growth. 
Table 3.5 A linear probability model explaining variation in stunting for different age groups, pooled 2005, 2010 and 2015-16 DHS

\begin{tabular}{|c|c|c|c|c|}
\hline \multirow{3}{*}{ Age range } & (1) & \multirow{2}{*}{$\begin{array}{c}\text { (2) } \\
24-59 \text { months }\end{array}$} & \multirow{2}{*}{$\begin{array}{c}\text { (3) } \\
\text { 6-23 months }\end{array}$} & \multirow{3}{*}{$\begin{array}{c}\text { (4) } \\
0-5 \text { months } \\
\text { Neonatal \& } \\
\text { early infancy }\end{array}$} \\
\hline & 0-59 months & & & \\
\hline & $\begin{array}{l}1000 \text { days \& post- } \\
1000 \text { days }\end{array}$ & Post-1000 days & 1000 days & \\
\hline \multirow[t]{2}{*}{ Child: female $(0 / 1)$} & $-0.051 * * *$ & $-0.035 * * *$ & $-0.090 * * *$ & -0.017 \\
\hline & $(0.006)$ & $(0.009)$ & $(0.010)$ & $(0.016)$ \\
\hline \multirow{2}{*}{ Child: birth interval<18m $(0 / 1)$} & $0.025 * * *$ & $0.048 * * *$ & -0.002 & -0.023 \\
\hline & $(0.009)$ & $(0.012)$ & $(0.016)$ & $(0.022)$ \\
\hline \multirow[t]{2}{*}{ Mother: teen pregnancy $(0 / 1)$} & $0.047 * * *$ & $0.041^{* * *}$ & $0.065 * * *$ & 0.033 \\
\hline & $(0.010)$ & $(0.014)$ & $(0.016)$ & $(0.024)$ \\
\hline \multirow{2}{*}{ Mother: Low BMI (0/1) } & $0.042 * * *$ & $0.053 * * *$ & 0.021 & $0.070 *$ \\
\hline & $(0.013)$ & $(0.017)$ & $(0.019)$ & $(0.036)$ \\
\hline \multirow[t]{2}{*}{ Mother: short $(<145 \mathrm{~cm})$} & $0.249 * * *$ & $0.283 * * *$ & $0.243 * * *$ & $0.112 *$ \\
\hline & $(0.022)$ & $(0.026)$ & $(0.037)$ & $(0.058)$ \\
\hline \multirow[t]{2}{*}{ Household: Asset score (0-10) } & $-0.018 * * *$ & $-0.028 * * *$ & -0.005 & $-0.014 *$ \\
\hline & $(0.003)$ & $(0.005)$ & $(0.006)$ & $(0.008)$ \\
\hline \multirow{2}{*}{ Household: Years sec. education } & $-0.003 * * *$ & $-0.003 * * *$ & $-0.003 * * *$ & 0.000 \\
\hline & $(0.001)$ & $(0.001)$ & $(0.001)$ & $(0.001)$ \\
\hline \multirow[t]{2}{*}{ Household: Improved water $(0 / 1)$} & $-0.015^{*}$ & $-0.022 * *$ & -0.007 & -0.000 \\
\hline & $(0.008)$ & $(0.011)$ & $(0.013)$ & $(0.019)$ \\
\hline \multirow[t]{2}{*}{ Household: Improved toilet (0/1) } & $-0.027 * *$ & $-0.030^{*}$ & -0.023 & -0.020 \\
\hline & $(0.012)$ & $(0.016)$ & $(0.019)$ & $(0.025)$ \\
\hline \multirow[t]{2}{*}{ Household: ANC blood tests $(0 / 1)$} & -0.009 & 0.004 & $-0.036^{* * *}$ & -0.010 \\
\hline & $(0.007)$ & $(0.010)$ & $(0.014)$ & $(0.020)$ \\
\hline \multirow[t]{2}{*}{ Village: Medical births $(0-1)$} & $-0.045 * *$ & $-0.056 * *$ & -0.041 & -0.009 \\
\hline & $(0.020)$ & $(0.026)$ & $(0.028)$ & $(0.038)$ \\
\hline \multirow{2}{*}{ Village: non-farm jobs $(0-1)$} & $-0.036^{*}$ & -0.042 & -0.042 & 0.037 \\
\hline & $(0.020)$ & $(0.027)$ & $(0.031)$ & $(0.042)$ \\
\hline Village: bednet use & $-0.082 * * *$ & $-0.087 * * *$ & $-0.069 * * *$ & $-0.104 * * *$ \\
\hline (if regional malaria $<20 \%$ ) & $(0.018)$ & $(0.023)$ & $(0.027)$ & $(0.035)$ \\
\hline Village: bednet use & $-0.042 *$ & -0.031 & $-0.087 * *$ & 0.047 \\
\hline (additional effect if malaria $>20 \%$ ) & $(0.025)$ & $(0.030)$ & $(0.037)$ & $(0.044)$ \\
\hline Region: ART knowledge (0-1) & -0.008 & 0.016 & 0.008 & -0.091 \\
\hline (if HIV <6\%) & $(0.077)$ & $(0.098)$ & $(0.114)$ & $(0.167)$ \\
\hline Region: ART knowledge (0-1) & $-0.073 * * *$ & -0.048 & $-0.102 * * *$ & $-0.131 * *$ \\
\hline (additional effect if HIV $>6 \%$ ) & $(0.026)$ & $(0.034)$ & $(0.037)$ & $(0.057)$ \\
\hline Region, survey \& age effects? & Yes & Yes & Yes & Yes \\
\hline Observations & 19,616 & 8,701 & 10,915 & 2,156 \\
\hline R-squared & 0.12 & 0.13 & 0.09 & 0.03 \\
\hline
\end{tabular}

Notes: This is a linear probability model with child stunting $(\mathrm{HAZ}<-2)$ as the dependent variable. $*, * *$ and $* * *$ refer to the $1 \%, 5 \%$ and $10 \%$ significance levels. See the main text for definitions of variables. 


\subsection{Robustness tests}

In this section we engage in a series of robustness tests that extend the core results reported above. Table 4.1 examines different specifications for malaria and HIV indicators, using a linear interaction between bednets and malaria prevalence and ART knowledge and HIV prevalence. This function assumes that the effects of bednets and ART knowledge are linearly dependent upon the prevalence of the disease, which makes sense provided that bednets and ART knowledge primarily influence stunting through reduction of the disease itself. These alternative interaction forms work well, with highly significant coefficients in most specifications. We again observe that bednets do not seem to explain much improvement in birth size, while ART knowledge is associated with stunting at younger ages but not older ages.

Table 4.1 A linear probability model explaining variation in stunting for different age groups as a function of linear interactions for malaria and HIV indicators, pooled 2005, 2010 and 2015-16 DHS

\begin{tabular}{|c|c|c|c|c|}
\hline Age range & $\begin{array}{c}(1) \\
0-59 \text { months } \\
1000 \text { days \& } \\
\text { post-1000 days }\end{array}$ & $\begin{array}{c}\text { (2) } \\
24-59 \text { months } \\
\text { Post-1000 days }\end{array}$ & $\begin{array}{c}(3) \\
\text { 6-23 months } \\
1000 \text { days }\end{array}$ & $\begin{array}{c}\text { (4) } \\
0-5 \text { months } \\
\text { Neonatal \& } \\
\text { early infancy }\end{array}$ \\
\hline Bednets*malaria prevalence ${ }^{\mathrm{a}}$ & $\begin{array}{c}-0.283^{* * *} \\
(0.084)\end{array}$ & $\begin{array}{c}-0.254^{* *} \\
(0.109)\end{array}$ & $\begin{array}{c}-0.430^{* * *} \\
(0.116)\end{array}$ & $\begin{array}{c}0.080 \\
(0.169)\end{array}$ \\
\hline ART knowledge* HIV prevalence & $\begin{array}{c}-0.966 * * * \\
(0.320)\end{array}$ & $\begin{array}{l}-0.569 \\
(0.396)\end{array}$ & $\begin{array}{l}-1.487 * * * \\
(0.442)\end{array}$ & $\begin{array}{c}-1.684 * * \\
(0.780)\end{array}$ \\
\hline $\begin{array}{l}\text { All other controls included } \\
\text { Region, survey \& age effects? }\end{array}$ & $\begin{array}{l}\text { Yes } \\
\text { Yes }\end{array}$ & $\begin{array}{l}\text { Yes } \\
\text { Yes }\end{array}$ & $\begin{array}{l}\text { Yes } \\
\text { Yes }\end{array}$ & $\begin{array}{l}\text { Yes } \\
\text { Yes }\end{array}$ \\
\hline $\begin{array}{l}\text { Observations } \\
\text { R-squared }\end{array}$ & $\begin{array}{c}21,775 \\
0.119\end{array}$ & $\begin{array}{c}12,108 \\
0.103\end{array}$ & $\begin{array}{l}7,306 \\
0.119 \\
\end{array}$ & $\begin{array}{l}2,361 \\
0.034 \\
\end{array}$ \\
\hline
\end{tabular}

In Table 4.2 we examine an alternative proxy for HIV programming, the share of adults who have been HIV tested in the past 12 months. For pregnant women and mothers, HIV testing is a prerequisite for qualification to PMTCT Option B+. In regressions (1) through (4) we use the interaction with the high HIV dummy variable. HIV testing predicts large and significant reductions in stunting reduction in the 0 59 and 6-23 month samples, and a large but non-significant association in the 0-5 month sample. In 
regressions (5) through (8) we use the linear interaction between HIV tests and HIV prevalence. The results here are more precisely estimated and significant for all samples, bar the older 24-59 month children.

Table 4.2 A linear probability model explaining variation in stunting for different age groups as a function of linear interactions for malaria and HIV indicators, pooled 2005, 2010 and 2015-16 DHS

\begin{tabular}{lcccc}
\hline Age range & $0-59$ months & $24-59$ months & $6-23$ months & $0-5$ months \\
& 1000 days \& & Post-1000 days & 1000 days & $\begin{array}{c}\text { Neonatal \& early } \\
\text { infancy }\end{array}$ \\
post-1000 days & &
\end{tabular}

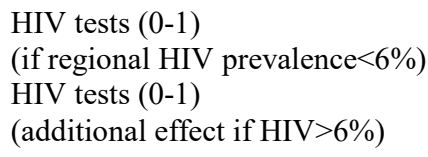

HIV tests*HIV prevalence ${ }^{a}$

All other controls included Region, survey \& age effects?

Observations

R-squared
(1)

$$
\begin{gathered}
-0.029 \\
(0.163) \\
-0.149 * * \\
(0.072)
\end{gathered}
$$

(5)

$$
\begin{gathered}
-2.139 * * * \\
(0.768) \\
\text { Yes } \\
\text { Yes } \\
\\
21,775 \\
0.119
\end{gathered}
$$

(2)

(3)

0.077
$(0.215)$
-0.121
$(0.096)$

(6)

$\begin{array}{cc}-3.505^{* * *} & -3.707 * * \\ (1.151) & (1.656)\end{array}$

Notes: This is a linear probability model with child stunting $(\mathrm{HAZ}<-2)$ as the dependent variable. *, ** and *** refer to the $1 \%, 5 \%$ and $10 \%$ significance levels. See the main text for definitions of variables. a. Adult HIV prevalence is measured for 2005 .

Next, we look at severe stunting (HAZ<-3) in Table 4.3. The pattern of results is similar to the stunting $(\mathrm{HAZ}<-2)$ results, with only a few notable differences. For severe stunting, improved water quality is significant, suggesting poor water may be associated with more infections and more severe forms of growth faltering. Bednet use continues to be significantly associated with stunting, but the additional impact in high-malaria regions is proportionally somewhat larger for severe stunting. Finally, ART knowledge continues to be robustly associated with severe stunting, similar to the patterns for $\mathrm{HAZ}<-2$. 
Table 4.3 A linear probability model explaining variation in severe stunting $(\mathrm{HAZ}<-3)$ for different age groups, pooled 2005, 2010 and 2015-16 DHS

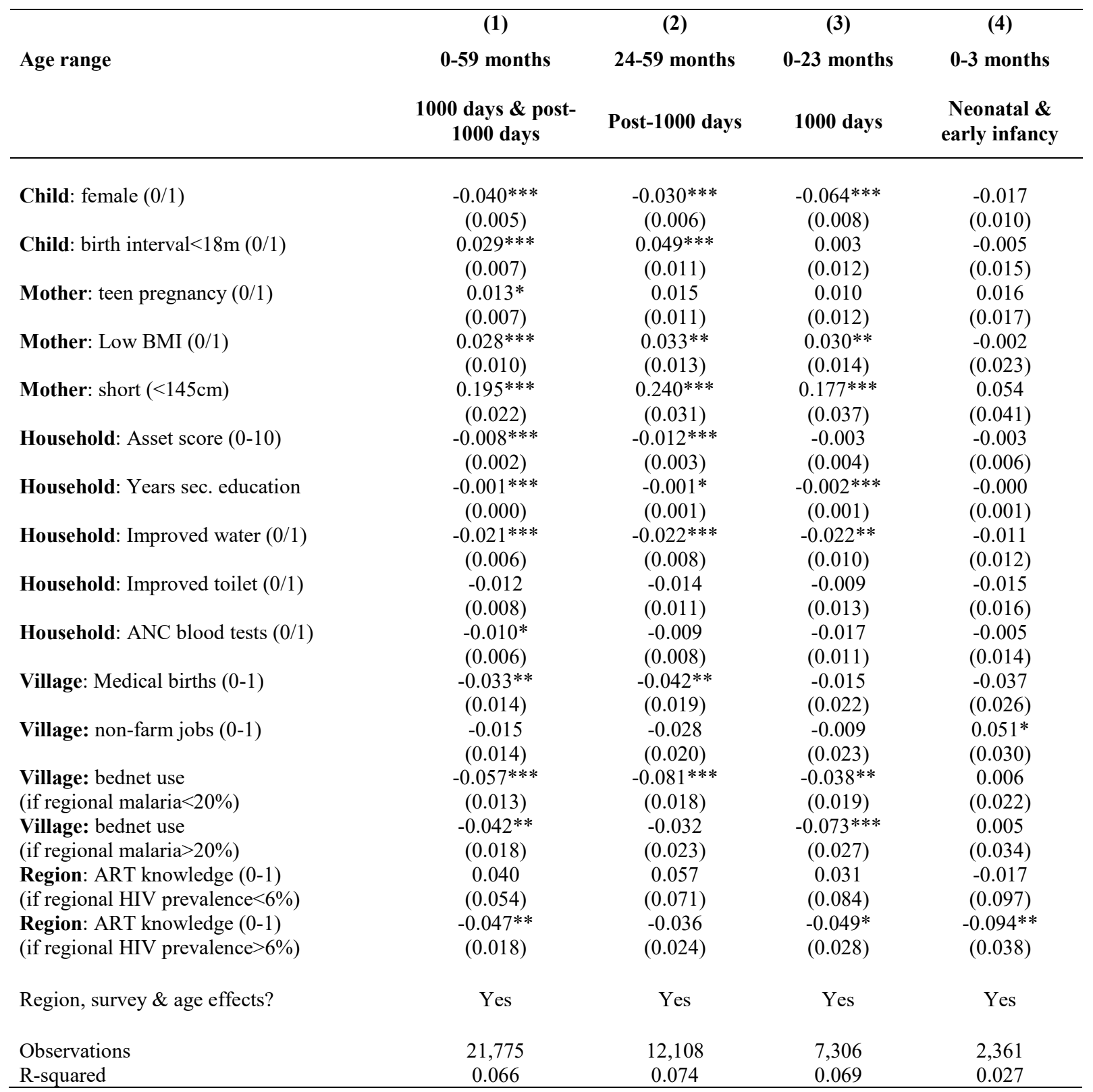

Notes: This is a linear probability model with child stunting $(\mathrm{HAZ}<-2)$ as the dependent variable. ${ }^{*}, * *$ and ${ }^{* * *}$ refer to the $1 \%, 5 \%$ and $10 \%$ significance levels. See the main text for definitions of variables

Table 4.4 reports results for rural and urban areas with a sample split between 0-23 and 24-59 months to preserve sufficiently large sample sizes. The smaller samples for urban areas result in less precision and more insignificant coefficients. Thus we make inferences more from the magnitude of the coefficients, rather than their statistical significance. In this regard the only notable difference is that improved water 
has a larger and more significant coefficient in the rural 24-59 month sample. Strikingly, the coefficients on malaria and ART knowledge are quite similar in rural and urban areas.

Table 4.4 A linear probability model explaining variation in stunting for rural and urban areas different age groups, pooled 2005, 2010 and 2015-16 DHS

\begin{tabular}{|c|c|c|c|c|}
\hline & (1) & (2) & (3) & (4) \\
\hline Age range & 24-59 months & 24-59 months & 0-23 months & 0-23 months \\
\hline Rural/urban? & Rural areas & Urban areas & Rural areas & Urban areas \\
\hline Child: female $(0 / 1)$ & $\begin{array}{c}-0.029 * * * \\
(0.010)\end{array}$ & $\begin{array}{c}-0.060 * * * \\
(0.018)\end{array}$ & $\begin{array}{c}-0.077 * * * \\
(0.010)\end{array}$ & $\begin{array}{c}-0.059 * * * \\
(0.020)\end{array}$ \\
\hline Child: birth interval<18m $(0 / 1)$ & $\begin{array}{c}0.055^{* * *} * \\
(0.014)\end{array}$ & $\begin{array}{l}-0.003 \\
(0.027)\end{array}$ & $\begin{array}{c}0.012 \\
(0.015)\end{array}$ & $\begin{array}{c}-0.123 * * * \\
(0.029)\end{array}$ \\
\hline Mother: teen pregnancy $(0 / 1)$ & $\begin{array}{c}0.042 * * \\
(0.017)\end{array}$ & $\begin{array}{c}0.040 \\
(0.027)\end{array}$ & $\begin{array}{c}0.057 * * * \\
(0.015)\end{array}$ & $\begin{array}{c}0.051 \\
(0.032)\end{array}$ \\
\hline Mother: Low BMI (0/1) & $\begin{array}{c}0.041^{* *} \\
(0.018)\end{array}$ & $\begin{array}{c}0.127 * * * \\
(0.042)\end{array}$ & $\begin{array}{c}0.026 \\
(0.020)\end{array}$ & $\begin{array}{c}0.055 \\
(0.042)\end{array}$ \\
\hline Mother: short $(<145 \mathrm{~cm})$ & $\begin{array}{c}0.260 * * * \\
(0.028)\end{array}$ & $\begin{array}{c}0.348 * * * \\
(0.062)\end{array}$ & $\begin{array}{c}0.206 * * * \\
(0.036)\end{array}$ & $\begin{array}{c}0.219 * * * \\
(0.075)\end{array}$ \\
\hline Household: Asset score $(0-10)$ & $\begin{array}{c}-0.034 * * * \\
(0.006)\end{array}$ & $\begin{array}{c}-0.022 * * * \\
(0.007)\end{array}$ & $\begin{array}{l}-0.005 \\
(0.006)\end{array}$ & $\begin{array}{l}-0.005 \\
(0.007)\end{array}$ \\
\hline Household: Years sec. education & $\begin{array}{c}-0.004 * * * \\
(0.001)\end{array}$ & $\begin{array}{c}-0.002 * * \\
(0.001)\end{array}$ & $\begin{array}{l}-0.002^{*} \\
(0.001)\end{array}$ & $\begin{array}{c}-0.004 * * * \\
(0.001)\end{array}$ \\
\hline Household: Improved water $(0 / 1)$ & $\begin{array}{c}-0.026^{* *} \\
(0.012)\end{array}$ & $\begin{array}{l}-0.003 \\
(0.031)\end{array}$ & $\begin{array}{l}-0.007 \\
(0.012)\end{array}$ & $\begin{array}{l}-0.019 \\
(0.031)\end{array}$ \\
\hline Household: Improved toilet $(0 / 1)$ & $\begin{array}{l}-0.025 \\
(0.020)\end{array}$ & $\begin{array}{l}-0.043 \\
(0.027)\end{array}$ & $\begin{array}{l}-0.029 \\
(0.020)\end{array}$ & $\begin{array}{l}-0.015 \\
(0.026)\end{array}$ \\
\hline Household: ANC blood tests $(0 / 1)$ & $\begin{array}{l}0.006 \\
(0.012)\end{array}$ & $\begin{array}{l}-0.001 \\
(0.020)\end{array}$ & $\begin{array}{c}-0.034 * * * \\
(0.013)\end{array}$ & $\begin{array}{l}-0.039 \\
(0.038)\end{array}$ \\
\hline Village: Medical births $(0-1)$ & $\begin{array}{c}-0.061 * * \\
(0.028)\end{array}$ & $\begin{array}{l}-0.090 \\
(0.073)\end{array}$ & $\begin{array}{l}-0.037 \\
(0.027)\end{array}$ & $\begin{array}{l}-0.094 \\
(0.070)\end{array}$ \\
\hline Village: non-farm jobs $(0-1)$ & $\begin{array}{l}-0.020 \\
(0.035)\end{array}$ & $\begin{array}{c}-0.182 * * * \\
(0.062)\end{array}$ & $\begin{array}{l}-0.031 \\
(0.035)\end{array}$ & $\begin{array}{l}-0.065 \\
(0.052)\end{array}$ \\
\hline Bednets*malaria prevalence ${ }^{a}$ & $\begin{array}{c}-0.236^{* *} \\
(0.117)\end{array}$ & $\begin{array}{c}0.194 \\
(0.292)\end{array}$ & $\begin{array}{c}-0.310^{* * * *} \\
(0.112)\end{array}$ & $\begin{array}{l}-0.389 \\
(0.279)\end{array}$ \\
\hline $\begin{array}{l}\text { ART knowledge* } \\
\text { women's HIV prevalence }\end{array}$ & $\begin{array}{l}-0.624 \\
(0.454)\end{array}$ & $\begin{array}{l}-0.695 \\
(0.791)\end{array}$ & $\begin{array}{c}-1.565 * * * \\
(0.469)\end{array}$ & $\begin{array}{l}-1.252 \\
(0.855)\end{array}$ \\
\hline Region, survey \& age effects? & Yes & Yes & Yes & Yes \\
\hline $\begin{array}{l}\text { Observations } \\
\text { R-squared }\end{array}$ & $\begin{array}{c}19,616 \\
0.12\end{array}$ & $\begin{array}{c}8,701 \\
0.13\end{array}$ & $\begin{array}{c}10,915 \\
0.09\end{array}$ & $\begin{array}{c}2,156 \\
0.03\end{array}$ \\
\hline
\end{tabular}

Notes: This is a linear probability model with child stunting $(\mathrm{HAZ}<-2)$ as the dependent variable. ${ }^{*}, * *$ and $* * *$ refer to the $1 \%, 5 \%$ and $10 \%$ significance levels. See the main text for definitions of variables. 
Finally, the Appendix to this chapter examines the external validity of our findings on the relationship between HIV programming proxies and child stunting by using a panel for subnational regions in all subSaharan African countries with the appropriate DHS datasets (24 in the context of PMTCT drug knowledge and 26 countries in the context of HIV testing). This larger Africa-wide panel involves sample sizes in excess of 600 region-years, as compared to just 78 region-years in the Tanzania-only panel used above. We estimate panel fixed effects regressions for this Africa-wide panel with different sets of controls: (1) fixed effects only; (2) fixed effects and time trends; and (3) fixed effects, time trends and control variables. The advantage of the Africa-wide panel is greater sample size and greater external validity, but there may be disadvantages too. In particular, the association between HIV programming proxies and actual treatment may vary across countries, and further work is needed to examine results for specific countries and regions.

Bearing these caveats in mind, the associations for ART knowledge are similar to those reported above but are more sensitive to the inclusion of time effects, while the results for HIV testing are somewhat more robust. For both indicators, the point estimates are similar in magnitude to the Tanzania-specific results reported above. Specifically, a 1 point increase in HIV testing or ART knowledge is associated with around a 0.07 point decrease in stunting, as opposed to a 0.08 point reduction for ART knowledge (unconditional on HIV prevalence) HIV testing in Tanzania. However, the Africa-wide results do not corroborate stronger impacts on younger children. In summary, a much larger Africa-wide panel confirms the presence of significant associations between HIV programming proxies and child stunting, but also warrants further research examining specific countries and regions.

\subsection{An extension to consider the role of child dietary diversity in reducing stunting}

A potential limitation of the regression analyses conducted above is that they do not include information on diets in any direct fashion. Dietary indicators were excluded from the analysis above because children's consumption of different foods was not included in the 2004-05 DHS, and was not well 
implemented in the 2010 DHS, with consumption of dairy products and grains being important omissions. However, dietary patterns and trends for the 2010 and 2015-16 DHS are reported in Table 4.5, based on DHS questions of the mother/caregiver about whether or not a child consumed specific foods in the past 24 hours. The available data for 2010 and 2015-16 shows no signs of increased consumption of nutrientrich foods, and perhaps some evidence of declining consumption of legumes/pulses, dark green leafy vegetables and roots/tubers (although the 2010 and 2015-16 were implemented at different times of the year, implying that seasonality issues could reduce the comparability of the two surveys).

The more complete data for 2015-16 can be used to look at patterns of consumption for individual foods, as well as the overall dietary diversity score out of the seven food groups listed in Table 4.5. The mean dietary diversity score is 2.96 out of 7 food groups, well below the WHO-recommended threshold of 4 food groups. Indeed, just under one third of children achieved minimum dietary diversity. Most children in this age range consume grains, roots or tubers, but just a third consumed Vitamin A-rich fruits or vegetables, and just over $20 \%$ consumed other fruits (e.g. bananas). Consumption of dark green leafy vegetables is common, however, as is pulse/legume consumption. Products that include dairy were less common. Roughly $20 \%$ of children in $2015 / 16$ consumed milk, yoghurt or cheese, and just $1.5 \%$ consumed infant formula. Fortified infant cereals - in principle, highly nutritious products - were consumed by just $12 \%$ of children. 
Table 4.5 Trends in consumption of different food groups in the past 24 hours in the 2010 and 201516 TDHS among children 6-23 months of age

2010 2015-16 Change

Dietary diversity score (0-7)

NA $\quad 2.96 \quad$ NA

Minimum dietary diversity (4/7 groups)

NA $\quad 32.7 \%$

NA

Individual food groups

(1) Grains, roots/tubers

Grains

$\begin{array}{ccc}\text { NA } & 86.6 \% & \text { NA } \\ \text { NA } & 82.7 \% & \text { NA } \\ 33.5 \% & 27.0 \% & -6.5 \% \\ 32.4 \% & 33.3 \% & 0.9 \% \\ 59.6 \% & 54.3 \% & -5.3 \% \\ 21.7 \% & 20.4 \% & -1.3 \% \\ 45.3 \% & 37.0 \% & -8.3 \% \\ \text { NA } & 19.9 \% & \text { NA } \\ 37.7 \% & 35.8 \% & -1.9 \% \\ 5.0 \% & 1.5 \% & -3.5 \% \\ 11.0 \% & 12.1 \% & 1.0 \%\end{array}$

Roots/tubers

(2) vit A-rich fruit/veg

(3) Dark green leafy veg

(4) Other fruit

(5) Legumes, pulses

(6) Dairy: Milk, yoghurt, cheese

$17.4 \%$

NA

NA

Source: Authors' estimates from the 2015-16 DHS. All statistics are nationally representative sample means.

Dietary diversity is plotted against child age in Figure 3.7, but separately for households with low, medium and high asset scores. Strikingly, there is no real difference between the poorest and middle asset groups, but children from the wealthiest group do approach minimum dietary diversity (MDD) (dietary diversity score $(\mathrm{DDS})=4)$ by around 12 months of age. 
Figure 3.7 Dietary diversity scores by child age for the poorest, middle and wealthiest households, children 6-23 months of age, 2015-16

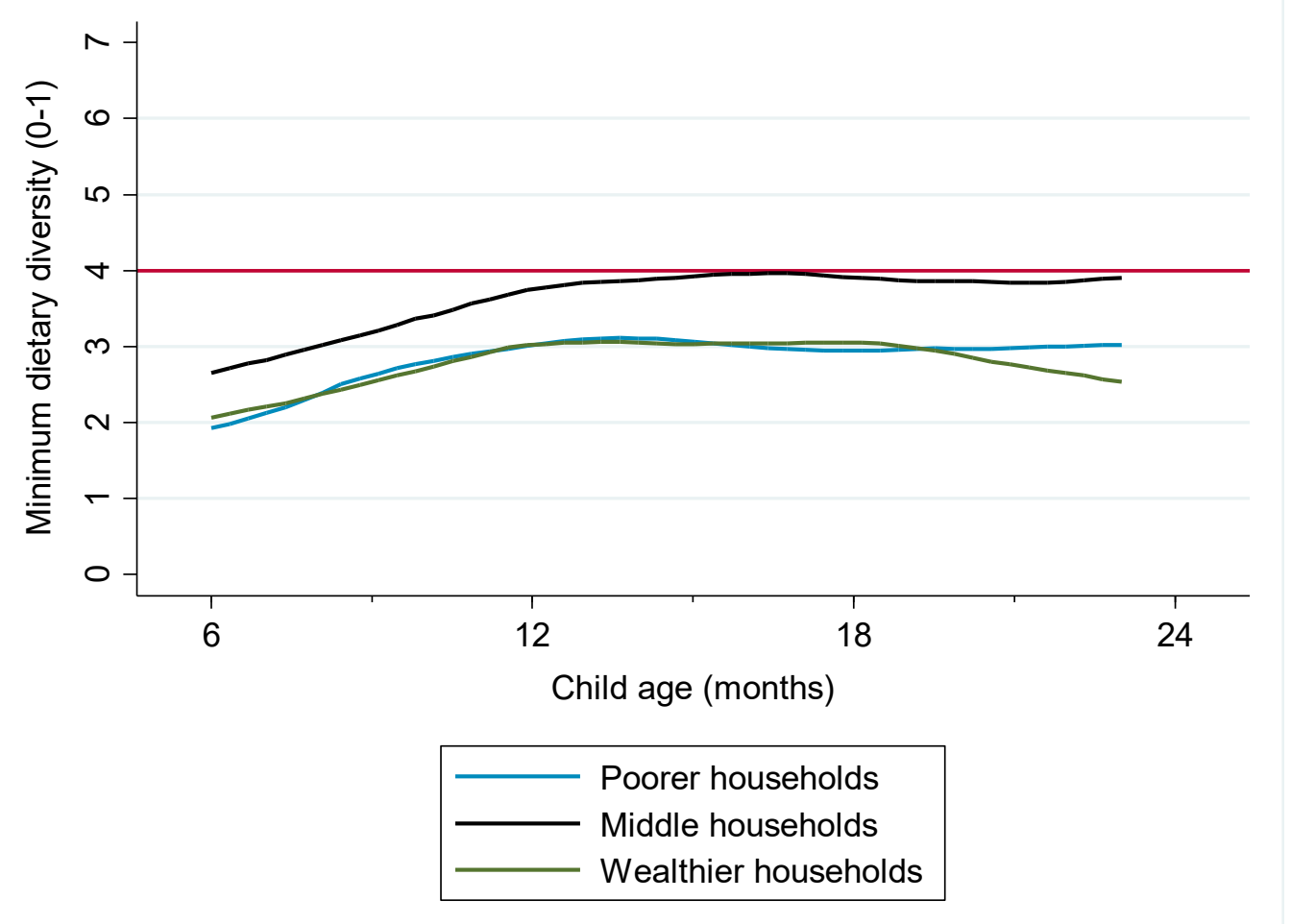

Table 4.6 examines dietary patterns by zones and rural-urban groups. DDS and MDD are highest in the Eastern zone (just under 50\%), followed by the Southern and Southern and Southwest highlands (just under 40\%), and Northern zone, and lowest in the Lake, Western, Central and Zanzibar zones. However, consumption of individual foods follows very diverse patterns. Vitamin A-rich fruit/vegetable consumption is highest in the Southern and Eastern zones. Dark green leafy vegetable consumption is relatively high everywhere except Zanzibar, while consumption of other fruit (e.g. bananas) is surprisingly low in almost all zones ( $20 \%)$. Legume/pulse consumption is $30-40 \%$ in most zones but low in the Southern and Zanzibar zones, while flesh food consumption is surprisingly high in most zones, seemingly because of fish. It is possible, however, that fish is consumed in small quantities, with limited impacts on nutrient intake. Dairy consumption is substantially higher in the Northern, Central and Eastern zones ( $40 \%$ ) but is less than $25 \%$ in other zones and very low in the Southern Highlands and Southern 
zones. Interestingly, the use of fortified infant cereals is high in the Eastern zone (30\%), possibly because of its more urban population, but uncommon elsewhere (10\% or less).

As one would expect, there are substantial rural-urban differences in child feeding patterns, particularly vitamin A-rich fruits/vegetables, eggs and flesh foods, and fortified infant cereals. There is no significant difference in dairy consumption, however, perhaps because some rural areas have high dairy consumption (e.g. the Northern zone). However, in terms of aggregate dietary diversity, urban children are much better off: almost half achieve MDD, as opposed to just a quarter of rural children. 
Table 4.6 Child food consumption patterns by zone and rural/urban status

\begin{tabular}{|c|c|c|c|c|c|c|c|c|c|c|c|}
\hline & DDS & MDD & $\begin{array}{l}\text { grains, } \\
\text { roots, } \\
\text { tubers }\end{array}$ & $\begin{array}{c}\text { vA-rich } \\
\text { fruit or } \\
\text { veg }\end{array}$ & $\begin{array}{c}\text { Dark } \\
\text { green } \\
\text { leafy veg }\end{array}$ & $\begin{array}{l}\text { Other } \\
\text { fruit }\end{array}$ & $\begin{array}{c}\text { Legumes, } \\
\text { pulses }\end{array}$ & $\begin{array}{l}\text { Eggs \& } \\
\text { flesh } \\
\text { foods }\end{array}$ & $\begin{array}{c}\text { Milk, } \\
\text { yoghurt, } \\
\text { cheese }\end{array}$ & $\begin{array}{c}\text { Infant } \\
\text { formula }\end{array}$ & $\begin{array}{c}\text { Fortified } \\
\text { infant } \\
\text { cereals }\end{array}$ \\
\hline Lake & 2.74 & $27.0 \%$ & $89.9 \%$ & $26.5 \%$ & $46.5 \%$ & $20.0 \%$ & $38.0 \%$ & $32.2 \%$ & $15.0 \%$ & $1.6 \%$ & $8.8 \%$ \\
\hline Western & 2.85 & $27.1 \%$ & $87.1 \%$ & $25.8 \%$ & $62.2 \%$ & $12.6 \%$ & $42.8 \%$ & $31.5 \%$ & $19.8 \%$ & $0.7 \%$ & $5.3 \%$ \\
\hline Northern & 2.96 & $35.8 \%$ & $77.2 \%$ & $29.1 \%$ & $42.6 \%$ & $28.1 \%$ & $29.9 \%$ & $42.4 \%$ & $43.4 \%$ & $1.3 \%$ & $9.1 \%$ \\
\hline Central & 2.79 & $26.5 \%$ & $87.9 \%$ & $21.7 \%$ & $54.7 \%$ & $22.9 \%$ & $31.7 \%$ & $21.1 \%$ & $33.8 \%$ & $1.1 \%$ & $8.9 \%$ \\
\hline Eastern & 3.51 & $46.8 \%$ & $88.1 \%$ & $53.9 \%$ & $59.6 \%$ & $21.6 \%$ & $41.6 \%$ & $42.7 \%$ & $17.7 \%$ & $4.2 \%$ & $30.1 \%$ \\
\hline S. Highlands & 3.24 & $39.1 \%$ & $90.6 \%$ & $47.1 \%$ & $72.8 \%$ & $20.7 \%$ & $39.5 \%$ & $37.8 \%$ & $6.6 \%$ & $0.0 \%$ & $10.6 \%$ \\
\hline SW Highlands & 3.04 & $37.7 \%$ & $78.6 \%$ & $35.5 \%$ & $65.3 \%$ & $21.1 \%$ & $41.5 \%$ & $37.9 \%$ & $13.4 \%$ & $0.0 \%$ & $12.8 \%$ \\
\hline Southern & 3.13 & $37.8 \%$ & $86.9 \%$ & $53.3 \%$ & $62.1 \%$ & $17.6 \%$ & $22.2 \%$ & $54.5 \%$ & $5.9 \%$ & $0.0 \%$ & $10.6 \%$ \\
\hline Zanzibar & 2.79 & $28.2 \%$ & $88.0 \%$ & $38.8 \%$ & $26.6 \%$ & $20.0 \%$ & $17.1 \%$ & $66.8 \%$ & $14.2 \%$ & $1.5 \%$ & $10.9 \%$ \\
\hline Rural & 2.76 & $26.8 \%$ & $86.2 \%$ & $27.9 \%$ & $54.0 \%$ & $18.0 \%$ & $35.3 \%$ & $30.2 \%$ & $20.1 \%$ & $1.0 \%$ & $6.5 \%$ \\
\hline Urban & 3.50 & $48.3 \%$ & $87.9 \%$ & $47.5 \%$ & $54.9 \%$ & $26.7 \%$ & $41.5 \%$ & $50.6 \%$ & $19.4 \%$ & $2.7 \%$ & $26.9 \%$ \\
\hline Total & 2.96 & $32.7 \%$ & $86.6 \%$ & $33.3 \%$ & $54.3 \%$ & $20.4 \%$ & $37.0 \%$ & $35.8 \%$ & $19.9 \%$ & $1.5 \%$ & $12.1 \%$ \\
\hline
\end{tabular}

Source: Authors' estimates from the 2015-16 DHS. All statistics are nationally representative sample means. 
In Table 4.7 we examine the potential importance of variation in child feeding patterns as a predictor of stunting, which is plausible given the steep growth faltering that takes place in the 6-23 month age range. Our set of control variables is identical to those in the regressions reported above, but the results pertain only to 2015-16. We first add MDD to this model. While the coefficient is negative and predictive of a 3 point decline in stunting, the coefficient is imprecisely estimated and not statistically significant from zero.

A recently published paper suggests that the direction of dietary diversification may matter, since ASFs may be more important for linear growth in early childhood because of their high density in multiple micronutrients as well as high-quality protein, fat and calories (Headey, Hirvonen and Hoddinott 2018). Regression (2) therefore specifies the individual food groups rather than MDD. While all the coefficients are negative, the only coefficient that is statistically different from zero is the one pertaining to dairy. Moreover, the coefficient is large in magnitude: daily dairy consumption predicts a 8.5-point reduction in the risk of stunting. This large coefficient on dairy is highly robust to alternative specifications, including controlling for village level maternal height, which may be a reasonably proxy for ethnic variations in height in Tanzania.

The importance of dairy for child growth is consistent with a range of research, including the aforementioned study on animal-sourced foods and stunting (where dairy also has the largest stunting reduction factor), as well as studies from Ethiopia (Hoddinott, Headey and Dereje 2014)), Uganda (Kabunga 2017), Rwanda (Rawlins et al. 2014) and Bangladesh (Choudhary and Headey 2018), and various experimental studies from both developed and developing countries (de Beer 2012; Iannotti et al. 2013). Biologically, dairy has the highest amino-acid correction factor of any food (i.e. its protein quality is exceptionally high), and is uniquely rich in calcium and B12, as well as other micronutrients (de Beer 2012; FAO 2013;). Dairy may also catalyze growth through insulin-like growth factor 1 . And as a complementary food, dairy is typically very palatable and familiar for infants and young children, and easy to prepare for time-constrained parents. The fact that only $20 \%$ of infants in Tanzania consumed 
dairy in 2015-16, despite the country having reasonable potential for domestic dairy production, suggests an opportunity to significant expand consumption in the future through both supply- and demand-side interventions.

Table 4.7 Linear probability models explaining variation in stunting among children 12-23 months as a function of minimum dietary diversity and consumption of individual foods groups, 2015-16 DHS

\begin{tabular}{lcc}
\hline & $\mathbf{( 1 )}$ & $\mathbf{( 2 )}$ \\
& $\mathbf{1 2 - 2 3}$ months & $\mathbf{1 2 - 2 3}$ months \\
Minimum dietary diversity & -0.030 & \\
vit A-rich fruit/veg & $(0.023)$ & -0.017 \\
Dark green leafy veg & & $(0.025)$ \\
& & -0.008 \\
Other fruit & & $(0.023)$ \\
& & -0.027 \\
Legumes, pulses & & $(0.025)$ \\
Eggs/flesh foods & -0.011 \\
Dairy & & $(0.022)$ \\
& & -0.011 \\
Region, survey \& age effects? & & $(0.026)$ \\
All other controls included? & Yes & $\left(0.085^{* * *}\right.$ \\
Observations & Yes & \\
R-squared & 1,795 & Yes \\
\hline
\end{tabular}

Notes: This is a linear probability model with child stunting $(\mathrm{HAZ}<-2)$ as the dependent variable. ${ }^{*}, * *$ and $* * *$ refer to the $1 \%, 55$ and $10 \%$ significance levels. The model controls for all the variables used in previous regression tables. 


\section{PAST AND FUTURE DRIVERS OF STUNTING REDUCTION}

In this section we use the linear decomposition at means method described in Section 2 to assess potential drivers of stunting reduction over 2005-2015, and to assess future drivers of stunting reduction to 2025 under an accelerated socio-economic development scenario.

\subsection{Using linear decompositions at means to assess drivers of stunting reduction over 2005-2015}

We now use the regression results from Table 3.5 and changes in sample means (survey-weighted) for each specific age group to implement a simple decomposition at means to explore the potential drivers of stunting reduction over 2004-05 to 2015-16 (Figure 5.1). Interpretation of this figure is as follows. First, each bar represents the retrospective change in stunting predicted by a particular set of similar factors. For example, using the Tanzanian results only, improvements in ART knowledge predict a 3.5 point decline in stunting in the 0-59 month group while the sum of ANC blood tests and medical births predict a 1.1 point decline. Second, the grey bars report unexplained changes in stunting, which is the actual change in stunting (around 13.4 points, depending on the sample) minus the sum of all the stunting changes predicted by each variable group.

Bearing these interpretations in mind, what do the results in Figure 5.1 suggest?

First, for the 0-59 month age group, we find that the overall model is quite successful in predicting total stunting reduction, accounting for $98 \%$ of the actual change in stunting. Much of the predicted decline in stunting stems from increases in ART knowledge (3.5 points), followed by bed net expansion (2.3 points), wealth and non-farm jobs (1.9 points), ANC blood tests plus medical births (1.0 points), and WASH (0.8 points).

Among older children (24-59 months) the predicted impacts of wealth and non-farm jobs are paramount, but among younger groups (0-23 months and 0-5 months) ART knowledge explains much of the stunting reduction, although the accuracy of these decompositions is somewhat reduced (indeed, the models overpredict the observed change). 
How should these decompositions be interpreted? First, it is important to bear in mind that these results are a useful means of interpreting potential drivers of change. The regression coefficients are measured with a certain degree of imprecision, and the true specification of the regression models is uncertain. Hence the decompositions should be regarded as somewhat conjectural. Second, bearing in mind the uncertainties involved, the results illustrate how developments in a range of different sectors appear to have been driving stunting reductions. They also show that even though some factors share important associations in multivariate regressions, their impacts in driving stunting reduction has been limited because of limited progress in these indicators over time. For example, maternal nutrition and education have not yet improved substantially, despite the regression results confirming their potential to reduce stunting. 
Figure 5.1 Decompositions of the predicted drivers of stunting reduction, 2004-05 to 2015-16

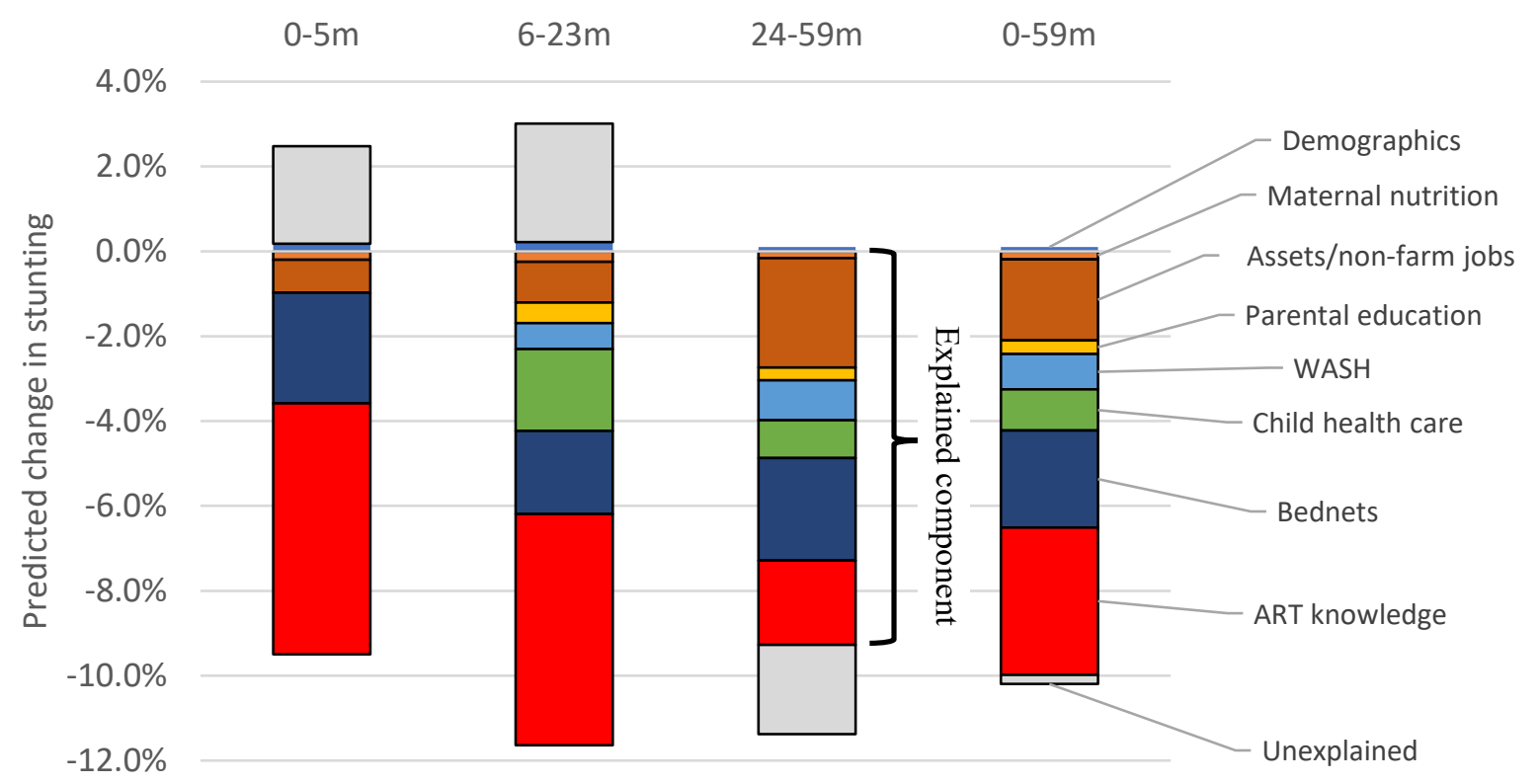

Notes: These decompositions for the first and third bars use the product of the regression coefficients based on the Tanzanian (TZA) sample from Table 3.6 and sample-specific changes in weighted means. The second and third bars are the same, but instead use the HIV test regression coefficients from Appendix Table A2.2, which pertain to a sample of all sub-Saharan African countries.

\subsection{Potential drivers of future stunting reduction in Tanzania: $2015-2025$}

The World Health Assembly (WHA) target on stunting is to reduce the number of stunted children by $40 \%$ by 2025 , while the far more ambitious SDG target of eliminating stunting by 2030 . Over 2005-2015 Tanzania made truly impressive progress in reducing stunting rates among pre-school children, although the evidence presented above suggests that virtually all of this reduction stems from improvement prenatal nutrition and birth size; postnatal growth faltering in 2015-16 was as precipitous as it was in 2004-05.

While it is crucial to continue improving maternal health and nutrition and antenatal care, in this section we concentrate on understanding how Tanzania can improve postnatal growth given the steep rising in stunting in early childhood, particularly from around 6 months onwards. To explore future scenarios, we use the regression results above to offer some conjectural predictions of how postnatal stunting rates 
could evolve under two different scenarios. For technical reasons, Table 5.1 focuses on stunting rates in the 24-59 month sample (i.e., the sample of children who have complete the first 1000 days of life and experienced full growth faltering over the 0-23 month window). As with the retrospective decompositions above, the predicted change in stunting from any given factor is just the assumed (imposed) changes in means over 2015-2025 multiplied by the regression coefficient for that variable (the "marginal effect").

In the first scenario the imposed changes in means over 2015-2025 stem from an assumption that the trends observed over 2004-05 to 2015-16 continue (Baseline scenario). In the second scenario we make the assumptions recorded in the last column of Table 5.1, which describe a much more accelerated pace of social and economic development, with growth in the asset score tripled, secondary education gains doubled, improved water reaching $90 \%$ of the population, medical facility births reaching $100 \%$, improved demographic incomes, and a tripling of dairy consumption among young children, from $20 \%$ to $60 \%$.

Not surprisingly, the results show that the 2005-2015 rate of progress is not sufficient to achieve meaningful reductions in postnatal growth faltering, with the 24-59 month stunting rate falling by just 5 percentage points from 37.3 to 32.3 . This is because $2005-2015$ saw increases in short-interval pregnancies, no change in adolescent pregnancies, and modest improvements in assets and secondary education. In contrast, the accelerated socioeconomic development scenario results in the $24-59 \mathrm{~m}$ stunting rate falling from $37.3 \%$ to $21.0 \%$, a 16.2 point reduction in stunting, implying a $44 \%$ reduction in the stunting rate, which approximates the WHA target of $40 \%$. 
Table 5.1 Alternative scenarios for improvements in postnatal growth via stunting changes in the 24-59 months age range

\begin{tabular}{|c|c|c|c|c|c|c|c|}
\hline & \multirow[b]{2}{*}{$\begin{array}{c}\text { 2004-05 } \\
\text { mean }\end{array}$} & \multirow[b]{2}{*}{$\begin{array}{c}\text { 2015-16 } \\
\text { mean }\end{array}$} & \multirow[b]{2}{*}{$\begin{array}{c}\text { Change in } \\
\text { mean }\end{array}$} & \multirow[b]{2}{*}{$\begin{array}{l}\text { Regression } \\
\text { Coefficient }\end{array}$} & \multicolumn{2}{|c|}{$\begin{array}{c}\text { Predicted change in stunting over } 2015-2025 \\
\text { (assumed change in means multiplied by regression } \\
\text { coefficient) }\end{array}$} & \multirow[b]{2}{*}{$\begin{array}{c}\text { Scenario } 2 \\
\text { assumptions }\end{array}$} \\
\hline & & & & & $\begin{array}{l}\text { Scenario 1: Baseline trends } \\
\text { continued } \\
(2005-15 \text { changes continue } \\
\text { over 2015-25) }\end{array}$ & $\begin{array}{l}\text { Scenario 2: Accelerated } \\
\text { socioeconomic development } \\
\text { (see next column) }\end{array}$ & \\
\hline $\begin{array}{l}\text { Asset score } \\
(0-10)\end{array}$ & 0.96 & 1.57 & 0.61 & -0.023 & $-1.40 \%$ & $-4.21 \%$ & Asset growth tripled \\
\hline $\begin{array}{l}\text { Increased dairy } \\
\text { consumption }\end{array}$ & NA & $20.50 \%$ & $0 \%$ & -0.085 & $0.00 \%$ & $-3.40 \%$ & $\begin{array}{l}\text { Dairy from } 20 \% \text { to } \\
60 \%\end{array}$ \\
\hline Medical births & $47.19 \%$ & $62.52 \%$ & $15.33 \%$ & -0.065 & $-1.00 \%$ & $-2.44 \%$ & Medical births to $100 \%$ \\
\hline Bed net coverage & $49.60 \%$ & $77.80 \%$ & $28.20 \%$ & -0.036 & $-0.44 \%$ & $-1.72 \%$ & Bed nets to $90 \%$ \\
\hline Maternal BMI score & 22.43 & 23.46 & 1.03 & -0.009 & $-0.93 \%$ & $-0.93 \%$ & Baseline trend \\
\hline $\begin{array}{l}\text { Non-farm } \\
\text { occupations }\end{array}$ & $27.95 \%$ & $48.61 \%$ & $20.66 \%$ & -0.035 & $-0.72 \%$ & $-0.92 \%$ & $\begin{array}{c}\text { Nonfarm occupation to } \\
75 \%\end{array}$ \\
\hline $\begin{array}{l}\text { Improved water } \\
\text { access }\end{array}$ & $45.23 \%$ & $56.25 \%$ & $11.02 \%$ & -0.021 & $-0.23 \%$ & $-0.92 \%$ & Improved water at $90 \%$ \\
\hline $\begin{array}{l}\text { Years secondary. } \\
\text { education }\end{array}$ & 1.74 & 2.68 & 0.94 & -0.003 & $-0.28 \%$ & $-0.85 \%$ & $\begin{array}{l}\text { Education gains } \\
\text { doubled }\end{array}$ \\
\hline Short birth intervals & $12.93 \%$ & $14.93 \%$ & $2.00 \%$ & 0.052 & $0.10 \%$ & $-0.39 \%$ & $\begin{array}{l}\text { Short birth interval } \\
\text { halved }\end{array}$ \\
\hline Teen pregnancies & $12.68 \%$ & $12.78 \%$ & $0.10 \%$ & 0.036 & $0.00 \%$ & $-0.23 \%$ & Teen pregnancy halved \\
\hline $\begin{array}{l}\text { Mother short } \\
(<145 \mathrm{~cm})\end{array}$ & $2.25 \%$ & $1.75 \%$ & $-0.50 \%$ & 0.29 & $-0.15 \%$ & $-0.15 \%$ & Baseline trend \\
\hline $\begin{array}{l}\text { Stunting among } \\
\text { children } 24-59 \mathrm{~m}\end{array}$ & $50.7 \%$ & $37.3 \%$ & & & $32.3 \%$ & $21.0 \%$ & \\
\hline
\end{tabular}


Although this scenario is feasible, achieving such dramatic improvements in these nutrition-sensitive sectors may well be difficult, and may point to the need to extend the scale and improve the effectiveness of nutrition-specific and nutrition-sensitive interventions, particularly those aimed at improving infant and young child feeding practices. Increasing dairy consumption may well depend on improving the affordability and safety of dairy products via supply-side interventions but may also require increasing parents' nutritional knowledge. Moreover, the weak association between other ASFs and stunting may well relate to small portion sizes as well as infrequent consumption. And, of course, dietary diversity matters for broader nutrition and health (e.g. improving immune system functioning), not just linear growth. Hence, there is considerable scope to strengthen the synergies between nutrition-sensitive and nutrition-specific sectors, particularly for common targets like improving diets. 


\section{CONCLUSIONS}

Tanzania has made impressive progress in addressing stunting over 2010-2016, especially. This study describes patterns of stunting improvement across the early lifecycle, explore zonal trends in stunting and its underlying determinants, and uses a systematic quantitative approach to explore associations between stunting and these determinants, and how the latter might explain stunting changes observed over time.

We find the following results.

First, Tanzania's rapid improvement in stunting over 2010-2016 stems partly from improvements in birth size and partly from reductions in postnatal growth faltering. The improvement in birth size is consistent with evidence of significant maternal weight gain in pregnancy. However, there are differences across locations. Improvements in rural areas tend to be more associated with improvements in birth size, whereas improvements in urban areas are more associated with reductions in stunting in the 6-24 month range. Likewise, in HIV prevalence regions stunting reduction seems to be associated with improvements in birth size, whereas in low HIV areas the association is more driven by postnatal growth improvements.

Second, Tanzania has experienced changes in multiple sectors, though many of the most impressive gains lie in the health sector, including antenatal and neonatal care, increased ART knowledge and HIV testing, and expansion of bednet use. Other gains were also evident, but more modest: improvements in assets and secondary education, as well as WASH, and significant increases in maternal BMI. However, results suggest there has been little improvement in household diets, which perhaps suggests that most of Tanzania's nutritional improvement stems from reduced rates of infection.

Third, improvement in birth size - which appears to be well captured by regressions in the $0-5$ month age range - is strongly associated with regional ART knowledge, and to a lesser extent with bednet coverage and household assets. The importance of HIV programming proxies is admittedly still uncertain, however, because Africa-wide regression yield results that are only partially consistent with Tanzania-specific results. However, the fact that HIV programming proxies are only significant in high HIV prevalence 
regions lends credence to the idea that PMTCT has been an important factor in improving birth size in Tanzania.

Fourth, for stunting reduction among all age groups (0-59 months), a wide range of factors were important, including household assets, ART knowledge, village bednet use, access to health care services, WASH, family planning outcomes and parental education. These results reinforce the highly multidimensional nature of undernutrition in early childhood, and the need for multisectoral strategies for addressing this problem.

Fifth, focusing only on the most recent 2015-16 round, we find that child dietary diversity in Tanzania is low, with less than a third of children meeting MDD. The relationship between MDD and stunting is negative but imprecise, whereas associations for individual foods emphasize the very strong negative association between stunting and child dairy consumption. While dietary diversity is important for closing multiple nutrient gaps and for achieving other nutrition and health objectives, the results suggest that increasing dairy consumption among children may be a very effective way of reducing stunting, consistent with published international research on this issue (Headey, Hirvonen and Hoddinott 2018).

Finally, we explored where future improvements in stunting must stem from. The dramatic improvements in birth size witnessed in recent years could be further strengthened with improvements in antenatal care and maternal diets, yet since most growth faltering now takes place in the postnatal period, various socioeconomic factors must play a greater role in improving child growth. We estimate that accelerated improvement in these factors will help Tanzania achieve the ambitious $40 \%$ reduction in stunting set by the WHA. However, those accelerations are themselves ambitious, and suggest that more expansive and more effective interventions, including ones that are nutrition specific (e.g., IYCF promotion) and nutrition-sensitive (e.g., gender, water and sanitation infrastructure) will be required to close the gap (Bhutta et al. 2013), particularly those that improve complementary feeding practices and reduce early childhood infections. A more comprehensive multisectoral approach to addressing the underlying determinants of undernutrition could well see Tanzania achieving even more rapid progress in the future. 


\section{REFERENCES}

Alderman, H., \& Headey, D. (2017). How Important is Parental Education for Child Nutrition? World Development, 94, 448-464. doi:http://dx.doi.org/10.1016/j.worlddev.2017.02.007

Alderman, H., \& Headey, D. (2018). The timing of growth faltering has important implications for observational analyses of the underlying determinants of nutrition outcomes. PLoS ONE, 13(4), e0195904. doi:10.1371/journal.pone.0195904

Bhatt, S., Weiss, D. J., Cameron, E., Bisanzio, D., Mappin, B., Dalrymple, U., . . Gething, P. W. (2015). The effect of malaria control on Plasmodium falciparum in Africa between 2000 and 2015. Nature, 526(7572), 207-211. doi:10.1038/nature15535

Bhattarai, Achuyt, Abdullah S. Ali, S. Patrick Kachur, Andreas Mårtensson, Ali K. Abbas, Rashid Khatib, Abdul-wahiyd Al-Mafazy et al. "Impact of artemisinin-based combination therapy and insecticide-treated nets on malaria burden in Zanzibar." PLoS medicine 4, no. 11 (2007): e309.

Bhutta, Z. A., Das, J. K., Rizvi, A., Gaffey, M. F., Walker, N., Horton, S., . . Black, R. E. (2013). Evidence-based interventions for improvement of maternal and child nutrition: what can be done and at what cost? The Lancet, 382(9890), 452-477.

Black, R. E., Victora, C. G., Walker, S. P., Bhutta, Z. A., Christian, P., de Onis, M., . . Uauy, R. (2013). Maternal and child undernutrition and overweight in low-income and middle-income countries. The Lancet, 382(9890), 427-451.

Black, R.E., Jackson, B.D., 2017. A Literature Review of the Effect of Malaria on Stunting. The Journal of Nutrition 147, 2163S-2168S.

Choudhury, S., and D. Headey. 2018. "Household dairy production and child growth: Evidence from Bangladesh." Economics \& Human Biology 30:150-161.

de Beer, H. 2012. "Dairy products and physical stature: a systematic review and meta-analysis of controlled trials." Economics \& Human Biology 10:299-309. 
Dutta, A., K. West Slevin, C. Barker and E. Leahy-Madsen. (2015). Maternal, newborn, and child health in Tanzania: costs and impacts of the one plan ii. Futures Group working paper.

Eisele, T. P., Larsen, D. A., Anglewicz, P. A., Keating, J., Yukich, J., Bennett, A., . . Steketee, R. W. (2012). Malaria prevention in pregnancy, birth weight, and neonatal mortality: a meta-analysis of 32 national cross-sectional datasets in Africa. Lancet Infect Dis, 12(12), 942-949. doi:10.1016/s1473-3099(12)70222-0

FAO. (2013). Milk and dairy products in human nutrition. Rome: Food and Agriculture Organisation (FAO).

Gamell, A., Luwanda, L. B., Kalinjuma, A. V., Samson, L., Ntamatungiro, A. J., Weisser, M., . . on behalf of the, K. S. G. (2017). Prevention of mother-to-child transmission of HIV Option B+ cascade in rural Tanzania: The One Stop Clinic model. PLoS ONE, 12(7), e0181096. doi:10.1371/journal.pone.0181096

Gillespie, S., \& van den Bold, M. (2017). Stories of Change in nutrition: An overview. Global Food Security, 13, 1-11. doi:https://doi.org/10.1016/j.gfs.2017.02.004

Gunther, I., \& Fink, G. (2010). Water, sanitation and children's health: evidence from 172 DHS surveys. Policy Research working paper no. WPS 5275. World Bank, Washington, DC.

Headey, D. (2014). An analysis of trends and determinants of child undernutrition in Ethiopia, 20002011. ESSP Working Paper No. 70. Addis Ababa.

Headey, D., \& Hoddinott, J. (2015). Understanding the Rapid Reduction of Undernutrition in Nepal, 2001-2011. PLoS ONE, 10(12), e0145738. doi:10.1371/journal.pone.0145738

Headey, D., \& Alderman, H. (2017). The relative prices of healthy and unhealthy foods in 175 countries. Paper presented at the Agriculture for Nutrition and Health Academy Week, Kathmandhu. Headey, D., \& Hoddinott, J. (2014). Understanding the rapid reduction of undernutrition in Nepal, 20012011 Discussion Paper No. 01384. Washington DC. 
Headey, D., Hoddinott, J., \& Park, S. (2016). Drivers of nutritional change in four South Asian countries: a dynamic observational analysis. Maternal \& Child Nutrition, 12, 210-218. doi:10.1111/mcn.12274

Headey, D., Hoddinott, J., Ali, D., Tesfaye, R., \& Dereje, M. (2015). The Other Asian Enigma: Explaining the Rapid Reduction of Undernutrition in Bangladesh. World Development, 66(0), 749-761. doi:http://dx.doi.org/10.1016/j.worlddev.2014.09.022

Headey, D.D., Hirvonen, K., Hoddinott, J.F., 2018. Animal sourced foods and child stunting. American Journal of Agricultural Economics 100, 1302-1319.

Heaver, R., \& Kachondam, Y. (2002). Thailand's National Nutrition Program: Lessons in Management and Capacity Development. World Bank HNP Discussion Paper. Washington, DC

Hirvonen, K. (2014). Measuring catch-up growth in malnourished populations. Annals of Human Biology, 41(1), 67-75. doi:10.3109/03014460.2013.827239

Hoddinott, J., Alderman, H., Behrman, J. R., Haddad, L., \& Horton, S. (2013). The economic rationale for investing in stunting reduction. Maternal \& Child Nutrition, 9, 69-82. doi:10.1111/mcn.12080

Hoddinott, J., D. Headey, and M. Dereje. 2015. "Cows, Missing Milk Markets, and Nutrition in Rural Ethiopia." The Journal of Development Studies 51:958-975.

Iannotti, L., E. Muehlhoff, and D. McMahon. 2013. "Review of milk and dairy programmes affecting nutrition." Journal of Development Effectiveness 5:82-115.

ICF International, \& USAID. (2017). Measure DHS Stat-Compiler. Retrieved November 22, from ICF International \& USAID http://www.statcompiler.com/

Irish Aid. (2013). Country Strategy Paper 2011-2015. Irish Aid.

Kabunga, N.S., S. Ghosh, and P. Webb. 2017. "Does ownership of improved dairy cow breeds improve child nutrition? A pathway analysis for Uganda." PLoS ONE 12:e0187816.

Kassebaum, N. J., Jasrasaria, R., Naghavi, M., Wulf, S. K., Johns, N., Lozano, R., Murray, C. J. L. (2014). A systematic analysis of global anemia burden from 1990 to 2010. Blood, 123(5), 615624. doi:10.1182/blood-2013-06-508325 
Kuecken, M., Thuilliez, J., \& Valfort, M. A. (2017). Disease and Human Capital Accumulation: Evidence from the Roll Back Malaria Partnership in Africa. Working Paper.

Lane, C.E., Bobrow, E.A., Ndatimana, D., Ndayisaba, G.F., Adair, L.S. (2019). Determinants of growth in HIV-exposed and -uninfected infants in the Kabeho Study. Maternal \& Child Nutrition 19, e12776.

Lartey, A., Marquis, G. S., Mazur, R., Perez-Escamilla, R., Brakohiapa, L., Ampofo, W., ... AduAfarwuah, S. (2014). Maternal HIV is associated with reduced growth in the first year of life among infants in the Eastern region of Ghana: The research to improve infant nutrition and growth (RIING) project. Maternal and Child Nutrition, 10(4), 604-616.

Le Roux, S., Abrams, E., Nguen, K., \& Myer, L. (2016). Clinical Outcomes of HIV-exposed, HIVuninfected Children in Sub-Saharan Africa. Tropical Medicine and International Health, 21(7), $829-845$.

Nkumama, I. N., O’Meara, W. P., \& Osier, F. H. A. (2017). Changes in Malaria Epidemiology in Africa and New Challenges for Elimination. Trends in Parasitology, 33(2), 128-140. doi:10.1016/j.pt.2016.11.006

Omoni, A. O., Ntozini, R., Evans, C., Prendergast, A. J., Moulton, L. H., Christian, P. S., \& Humphrey, J. H. (2017). Child Growth According to Maternal and Child HIV Status in Zimbabwe. Pediatric Infectious Disease Journal, 36(9), 869-876.

Rawlins, R., S. Pimkina, C.B. Barrett, S. Pedersen, and B. Wydick. 2014. "Got milk? The impact of Heifer International's livestock donation programs in Rwanda on nutritional outcomes." Food Policy 44:202-213.

Unger, H. W., Ashorn, P., Cates, J. E., Dewey, K. G., \& Rogerson, S. J. (2016). Undernutrition and malaria in pregnancy - a dangerous dyad? BMC Med, 14(1), 142. doi:10.1186/s12916-016-06952

UNICEF. (1990). Strategy for Improved Nutrition of Children and Women in Developing Countries. Retrieved from New York: 
Victora, C. G., de Onis, M., Curi Hallal, P., Blössner, M., \& Shrimpton, R. (2009). Worldwide Timing of Growth Faltering: Revisiting Implications for Interventions. Pediatrics, 2010(125), 473-480.

Volmink, J. A., \& Marais, B. J. (2008). HIV: mother-to-child transmission. BMJ Clinical Evidence, 2008, 0909.

World Bank. (1999). Tanzania: Social Sector Review. Washington, DC: The World Bank.

World Bank. (2017). World Development Indicators Online. from The World Bank http://devdata.worldbank.org/dataonline/

World Health Organization (WHO). 1999. "Rolling Back Malaria". In The World Health Report 1999. Pages 49 - 63. http://www.who.int/whr/1999/en/whr99 ch4_en.pdf

WHO, 2006. WHO Child Growth Standards based on length/height, weight and age. Acta Paediatrica Supplement 450, 76-85. 


\section{ALL IFPRI DISCUSSION PAPERS}

All discussion papers are available here

They can be downloaded free of charge

INTERNATIONAL FOOD POLICY RESEARCH INSTITUTE www.ifpri.org

\section{IFPRI HEADQUARTERS}

1201 Eye Street, NW

Washington, DC 20005 USA

Tel.: +1-202-862-5600

Fax: +1-202-862-5606

Email: ifpri@,cgiar.org 